\title{
Uncertainty analysis and validation of the estimation of effective hydraulic properties at the Darcy scale
}

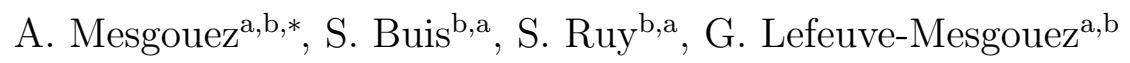 \\ ${ }^{a}$ Université d'Avignon et des Pays de Vaucluse, UMR1114 EMMAH, Faculté des \\ Sciences, F-84000 Avignon, France \\ ${ }^{b}$ INRA, UMR1114 EMMAH, F-84914 Avignon, France
}

\begin{abstract}
The determination of the hydraulic properties of heterogeneous soils or porous media remains challenging. In the present study, we focus on determining the effective properties of heterogeneous porous media at the Darcy scale with an analysis of their uncertainties.

Preliminary, experimental measurements of the hydraulic properties of each component of the heterogeneous medium are obtained. The properties of the effective medium, representing an equivalent homogeneous material, are determined numerically by simulating a water flow in a three-dimensional representation of the heterogeneous medium, under steady-state scenarios and using its component properties. One of the major aspects of this study is to take into account the uncertainties of these properties in the computation and evaluation of the effective properties. This is done using a bootstrap method.
\end{abstract}

\footnotetext{
* Corresponding author

Email addresses: arnaud.mesgouez@univ-avignon.fr (A. Mesgouez), sbuis@avignon.inra.fr (S. Buis), ruy@avignon.inra.fr (S. Ruy), gaelle.mesgouez@univ-avignon.fr (G. Lefeuve-Mesgouez)
} 
Numerical evaporation experiments are conducted both on the heterogeneous and on the effective homogeneous materials to evaluate the effectiveness of the proposed approach. First, the impact of the uncertainties of the component properties on the simulated water matric potential is found to be high for the heterogeneous material configuration. Second, it is shown that the strategy developed herein leads to a reduction of this impact. Finally, the adequacy between the mean of the simulations for the two configurations confirms the suitability of the homogenization approach, even in the case of dynamic scenarios.

Although it is applied to green roof substrates, a two-component media composed of bark compost and pozzolan used in the construction of buildings, the methodology proposed in this study is generic.

Keywords: effective hydraulic parameters, heterogeneous porous media, uncertainty estimation, green roof substrate, the Richards equation, bootstrapping method

\section{Introduction}

At the Darcy scale, heterogeneous media can be represented in different ways. Basically, a heterogeneous medium can be modeled either using 4 a spatial distribution of properties inside a single unit (Khaleel et al., 2002; 5 Mantaglou and Gelhar, 1987; Russo, 1992; Vogel et al., 2010; Yeh et al., 1985 6 among others), or using a patchwork of homogeneous sub-units (Bechtold et al., 2012; Samouëlian et al., 2011; Wildenschild and Jensen, 1999a). A combination of both approaches (Javaux and Vanclooster, 2006a; Zhang et al., 9 2010) can also be used. A detailed description of the various representations 
can be found in the reviews of Renard and de Marsily (1997) or Vereecken et al. (2007). In our study, we refer to the second way of representation, based on a patchwork of homogeneous sub-units.

The determination of these sub-units is a difficult task and can be performed in different ways. Invasive methods for delineating three-dimensional natural soil units include pedological or geological observations (Bierkens and van der Gaast, 1998; Javaux and Vanclooster, 2006b ; Ma et al., 2010 ; Samouëlian et al., 2011) and dye tracing experiments (Javaux and Vanclooster, 2006a). Non invasive methods such as two-dimensional electrical resistivity tomography (Besson et al., 2004; Tabbagh et al., 2000) or threedimensional x-ray computed assist tomography (Duliu, 1999) can also be used. The alternative approach is to work on remolded porous media, such as calibrated sands, to precisely control the heterogeneity pattern and the distribution of hydraulic properties (Bechtold et al., 2012; Danquigny and Ackerer, 2005; Wildenschild and Jensen, 1999b). In our study, we refer to this last way of representation.

When working on a patch of homogeneous sub-units, each component of the heterogeneous medium is considered as a single-phase continuum described by macroscopic laws (the Darcy law and the Richards equation). Water flow simulation or water balance computation can then be performed on the elementary volume by using a numerical solver of the Richards equation accounting for the spatial heterogeneity of the hydraulic properties. The spatial structure must be known to distribute the hydraulic properties of each material on the volume of interest. Moreover, solving the Richards equation can be tedious, in particular for three-dimensional flow in complex 
heterogeneous geometry (Herbst et al., 2008). A simpler approach consists in replacing the explicit three-dimensional structure of the heterogeneous volume by a homogeneous medium, wherein the properties of the homogenized medium take into account the hydraulic properties of each material, in such a way that simulations conducted on both domains yield similar water fluxes under identical boundary conditions (Samouëlian et al., 2011). Such medium properties are then called effective properties. The effective medium is still obtained at the Darcy scale. The homogenization approach can then be considered as an upscaling technique from a continuum scale to a larger continuum one.

Due to the recent advances in computing capabilities, numerical approaches are now widely used for the determination of effective properties (Samouëlian et al., 2007; Vogel et al., 2010). The objective of this study is to contribute to the evaluation of this approach. Several authors noted that simulations conducted using effective parameters may differ from simulations conducted with spatially distributed parameters (Vogel et al., 2008; Vogel et al., 2010) or from experimental data measured on heterogeneous media (Wildenschild and Jensen, 1999b). In addition, though several authors estimated the effective hydraulic properties of various natural soils (Javaux and Vanclooster, 2006a; Samouëlian et al.,2011; Vogel and Roth, 1998), no study, to our knowledge, integrates a complete uncertainty evaluation of this procedure. The process of estimating the hydraulic properties of a given material includes however various sources of uncertainties (Mohrath et al., 1997; Peters and Durner, 2008; see also section 2.2 of this article). These uncertainties significantly affect the results of numerical simulations (Chris- 
tiaens and Feyen, 2001; Coppola et al., 2009; Pan et al., 2009) and thus the estimation of effective properties or their evaluation using dynamic scenarios. In this study, we propose to estimate the uncertainties on the effective hydraulic properties of a real heterogeneous material, and to evaluate these properties and the impact of their uncertainties by comparing simulations of a dynamic process using either the heterogeneous medium or the effective homogeneous material.

The methodology proposed in this article is generic. It is applied here to green roof substrates, hereafter called substrate or complex substrate. It is a composite of compressible materials, namely organic matter (bark compost) used as fertilizer, and of aggregates of volcanic rock (pozzolan) used as rigid skeleton. This two-component material is considered to serve a number of beneficial purposes that can help in the management of various environmental problems, such as the reduction of air pollution or of the carbon footprints of cities (Yang et al., 2008), the improvement of storm water management (Carter and Jackson, 2007) and the improvement of energy efficiency in buildings (Ouldboukhitine et al., 2012).

\section{Materials and Methods}

The methodology followed in this study is sketched up in Fig. 1. The heterogeneous material under study is composed of a combination of bark compost and pozzolan. In the first step, water retention and hydraulic conductivity are measured for both materials using ad hoc experimental procedures for different water matric potential and on different samples. The hydraulic properties are then estimated by fitting parametric models to the 
experimental data. The uncertainties on these properties are estimated using a bootstrap method.

In the second step, effective hydraulic parameters are determined by simulating numerically the water flow in a three-dimensional representation of the heterogeneous material taking into account the uncertainties on the hydraulic properties of each constitutive materials and on the spatial distribution of the materials using Monte Carlo random sampling. The effective hydraulic properties and associated uncertainties are then estimated in the same way as for single material properties but using the simulated water retention and conductivity values.

Finally, in the third step, the reliability of the computed effective hydraulic properties is evaluated. Simulations of the evolution of water matric potential versus time are performed under dynamic scenarios with the effective medium and the heterogeneous material taking into account the uncertainties on their hydraulic properties and the spatial distribution of the materials. Their mean behaviors are cross-checked and the impact of the uncertainties is compared.

The following subsections give details on the models used for fitting hydraulic properties, the methodology followed for estimating their uncertainties, the numerical configuration of the water flow simulations, the method used for computing the effective properties and the evaluation of the homogenization approach. The experimental procedures used for measuring water retention and hydraulic conductivity for bark compost and pozzolan and the specific $\mathrm{C}++$ parallelized code used for solving the Richards equation are described in Appendix A and B respectively. 


\subsection{Models of hydraulic properties}

The van Genuchten model (van Genuchten, 1980) is fitted to the experimental data to obtain the hydraulic properties, from which we deduce the water retention curve, i.e. the relation between $\theta(h, \mathbf{x}, t)\left[L^{3} L^{-3}\right]$, the volumetric water content, and $h(\mathbf{x}, t)[L]$, the water matric potential, as follows

$$
\theta(h, \mathbf{x}, t)=\theta_{r}(\mathbf{x})+\left(\theta_{s}(\mathbf{x})-\theta_{r}(\mathbf{x})\right) \times\left[1+\left(\frac{h(\mathbf{x}, t)}{h_{e}(\mathbf{x})}\right)^{n}\right]^{-m}
$$

where $\mathbf{x}[L]$ are the spatial coordinates, $t[T]$ is the time, $\theta_{r}\left[L^{3} L^{-3}\right]$ is the residual volumetric water content, $\theta_{s}\left[L^{3} L^{-3}\right]$ is the water content at saturation, $h_{e}[L]$ is a scale parameter, $n[-]$ and $m[-]$ are shape parameters with $m=1-1 / n$.

The Mualem-van Genuchten model (van Genuchten, 1980) is fitted to the experimental data to obtain the hydraulic properties, from which we deduce the hydraulic conductivity curve, i.e. the relation between $K(h, \mathbf{x}, t)\left[L T^{-1}\right]$, the hydraulic conductivity, and $h(\mathbf{x}, t)$, the matric water potential, as follows

$$
K(h, \mathbf{x}, t)=K_{\text {Sat }}(\mathbf{x}) \cdot \frac{\left[1-\left(h(\mathbf{x}, t) / h_{e}(\mathbf{x})\right)^{n-1} \times\left(1+\left(h(\mathbf{x}, t) / h_{e}(\mathbf{x})\right)^{n}\right)^{-m}\right]^{2}}{\left[1+\left(h(\mathbf{x}, t) / h_{e}(\mathbf{x})\right)^{n}\right]^{m / 2}}
$$

(1)




\subsection{Uncertainty evaluation methodology}

As already mentioned, various approximations and errors can be sources of uncertainty when estimating the hydraulic properties of a material. The following typology is proposed to classify these sources of uncertainty:

1. Variability in the properties of samples: owing to the variability of the material samples used in some experiments, or due to the fact that some experiments result in the destruction of the samples, measurements of the properties of a material are often performed on replicates. The number of replicates may have a significant impact on the estimation of the hydraulic properties of a material depending on the level of their individual variability.

2. Errors in experimental data due to (i) approximations used for size characterization, weight or density measurements, sensor locations, and to (ii) digitalization errors associated with digital data-loggers. These errors can propagate in the estimation of the hydraulic properties as noted by Tamari et al. (1993).

3. Model errors due to inadequate model assumptions. Vogel et al. (2010) showed in a numerical case study that the validation of the homogenization process requires a highly flexible hydraulic law model.

4. Fitting errors: in the presence of a low number of experimental data, the level of uncertainty of the obtained parameters using parametric models fitted to the experimental data may be very high, even in case of slight variability in the properties of the samples and of slight measurement errors. 
In our study the bootstrap method (Efron and Tibshirani, 1994; Manly, 2006) is used to estimate the resulting uncertainties on the hydraulic properties. This method can be used to estimate a statistic without it being biased, to evaluate the accuracy of this estimation and/or to build confidence intervals for this statistic. In the present study, the statistics computed are the parameters of the models describing the hydraulic properties. The principle of non-parametric bootstrapping is briefly described below.

Let us consider the observation sample obtained for the computation of the statistic, in our case a set of water retention or conductivity measurements. These observations must be independent and identically distributed to ensure the convergence of the method. This is the case for almost all configurations in our study and when it is not completely the case (pozzolan and compost conductivity measurements), it has been checked that the presence of dependencies does not significantly influence the results. $N$ artificial samples, of the same size as the original observation sample, are created by sampling in it with replacement. They are called bootstrap samples. The value of the statistic is computed for each one of these bootstrap samples. Its probability distribution is then approximated using the histogram of its $N$ computed values. In other words, the actual variability of the statistic is estimated using the observed variability of all the samples obtained by resampling. In our case, the uncertainty distributions of the estimated hydraulic properties are thus approximated by fitting the models $N$ times on bootstrap samples of the observations. The number of bootstrap samples used is always set to $N=500$. It has been verified that the results obtained (mean and standard deviation of the parameters estimated) are stable for 
this number (variation of only a few percent).

Though this methodology does not take into account the problem of model error, of large biases or of samples non representative of the population, it produces accurate descriptions of uncertainties under reasonable assumptions and a lower bound of the actual uncertainty level. Compared to the maximum likelihood approach, that is classically used for model fitting and allows estimating a covariance error matrix for the parameters, the bootstrap approach has the advantage of being free of hypothesis concerning the linearity of the model and thus allows estimating non-gaussian error distribution for the parameters. The joint distribution estimated contains the possible dependencies between parameter values resulting from the calibration process. Finally bootstrap method directly gives a sample of this distribution that represents the parameter uncertainties and their dependencies. This sample can then easily be used for Monte Carlo uncertainty propagation. The bootstrap method is particularly well suited in our case since numerous replicated observations are available and the models to fit are simple. In case of time-expensive models or numerous parameters, alternative methods could be used (see for example Brunner et al., 2012).

\subsection{Configuration for Richards equation computation}

Numerical simulations are performed on a typical soil core to compute the effective parameters at the Darcy scale and to evaluate the reliability of these parameters under dynamic scenarios. In our case, the green roof substrate is represented by a cylinder with the following dimensions: height $=7 \mathrm{~cm}$ and diameter $=15 \mathrm{~cm}$. The cylinder is discretized with 507,553 nodes and 3, 216, 152 elements. The average length of each edge is approximately 1.5 
mm. As mentioned in Appendix A, the pozzolan aggregate size distribution is the following: $2 / 3$ of the pozzolan aggregate diameters ranged from 3 to 6 $\mathrm{mm}$, and $1 / 3$ from 7 to $15 \mathrm{~mm}$. A specific algorithm is developed to model the geometry and distribution of pozzolan. This algorithm uses a mesh of the cylinder obtained by GMSH (http://geuz.org/gmsh/). Aggregates of pozzolan are then created by randomly selecting a seed represented by an element of the mesh. The volume of the aggregate is randomly chosen from the known aggregate diameter distribution and under the hypothesis that the pozzolan aggregates are spheric. The irregular aggregates are then iteratively built by randomly selecting and aggregating adjacent elements of the growing irregular seeds until the required aggregate volumes are reached. Fig. 2 shows a typical mesh used in the simulations. Initial and boundary conditions are specific to the problem solved (effective parameter computation or evaluation under a dynamic scenario) and are detailed in subsections 2.4 and 2.5.

\subsection{Effective parameter computation}

The effective parameters are obtained using a steady-state flow simulation, as explained in Samouëlian et al. (2007) or Samouëlian et al. (2011). The interval under consideration is $\left[-10^{2},-10^{-2}\right] \mathrm{m}$, which corresponds to the validity range of the numerical code. The same water matric potential, a Dirichlet type condition, is applied to the upper and the lower boundaries of the cylinder. This leads to a constant water matric potential throughout the medium in the case of homogeneous materials. The vertical boundaries are impermeable. To obtain the hydraulic conductivity, the calculated water flux is divided by the surface of the upper boundary. In the case of a heterogeneous medium, the same configuration leads to an almost constant 
water matric potential in the medium, and the so-called effective hydraulic conductivity of the composite substrate is equal to the average water flux density across the horizontal boundaries. The water retention is calculated for each element with a simple arithmetic average. These conductivity and water retention values are relative to the matric potential value set at the boundaries, even if local gradients of matric potential are present within the heterogeneous medium. The computation is thus repeated for several water matric potential values to fit the conductivity and retention curves.

To take into account uncertainties in the hydraulic properties of pozzolan and compost, the computation of the effective hydraulic conductivity and water retention for each water matric potential value is repeated for a single set of component properties randomly sampled from their uncertainty distributions. The sizes, shapes and spatial distribution of the pozzolan aggregates in the mesh are different for each repetition and are generated randomly as explained in section 2.3. The sampling of pozzolan and compost properties is performed independently for each water matric potential value.

The choice of the number and values of water matric potential points, and the number of replicates for each water matric potential value, directly affects the computational cost of the estimation of the effective properties and of their level of uncertainty. An optimal experimental design technique is used to guide these choices. A D-optimal criterion (Atkinson and Donev, 1992) is computed to fit the Mualem-van Genuchten and the van Genuchten models. The aim is to minimize the D-criterion, which is the determinant of the error covariance matrices of the models parameters. Several contrasted water matric potential distributions of points are considered in the bounded 
interval $\left[-10^{2},-10^{-2}\right] \mathrm{m}$ : regular distribution, log-regular distribution and quantiles of beta distributions to concentrate points on the left, the right or the center of the interval. The log-regular distribution appears to be the best compromise with respect to the criterion values computed for both models for a number of water matric potential values. Then, an optimization of the ratio between the number of water matric potential points and the number of replicates, for a given computational cost corresponding to 200 simulations, is computed with this log-regular distribution. In the end, the D-optimal design, among those tested, is a log-regular repartition of 4 water matric potential points with 50 replicates. For the Mualem-van Genuchten model fit, prior information from the Wiener bounds on $K_{\text {sat }}$ is considered to regularize the fitting problem due to the lack of data at $h=0 \mathrm{~m}$.

\subsection{Evaluation of the homogenization approach}

In order to reduce the impact of non-equilibrium water flow that may appear during dynamic scenarios (Vogel et al., 2008), we use an evaporation scenario to evaluate the homogenization approach because it was noticed that non-equilibrium water flow is more likely to be produced by sharp moisture front obtained during the infiltration process (Roth, 2008). A time-variable flux of evaporation ranging from $1.9 \mathrm{~mm} \mathrm{~d}^{-1}$ to $0.9 \mathrm{~mm} \mathrm{~d}^{-1}$ is applied to the top surface of the soil core at $z=0$. This evaporation flux is imposed as a Neumann boundary condition at the core surface. The values of the timevariable flux are representative of usual experimental conditions when using the Wind evaporation method. The other boundaries are impermeable. The initial condition is that of a quasi-saturated medium with $h(\mathbf{x}, t=0)=-9.5$ $\mathrm{cm}$ for all depths. For $h=-100 \mathrm{~m}$, the flux type boundary conditions are 
switched to Dirichlet conditions. The simulations last 10 days to ensure a strong evaporation and a switch from flux to Dirichlet conditions at the upper part of the soil core.

200 simulations are performed for the two-component configuration and for the equivalent homogeneous material, by randomly sampling the corresponding hydraulic properties in their uncertainty distributions and the sizes, shapes and spatial distribution of the pozzolan aggregates. For each simulation, 4 sets of 5 points are considered to monitor the matric potential. They are taken for $z=0,-0.2,-1.1$ and $-4.8 \mathrm{~cm}$, respectively. For each depth, the arithmetic mean values of matric potential are computed on the 5 points. The distribution of these mean values obtained for the two-component configuration is then compared to the distribution obtained for the equivalent homogeneous configuration at each depth.

\section{Results and Discussion}

\subsection{Hydraulic properties and associated uncertainties of individual materials}

The water retention measurements of pozzolan are widely spread near saturation (Fig. 3a). High standard deviations for water content, $0.13 \mathrm{~m}^{3}$ $\mathrm{m}^{-3}$ to $0.14 \mathrm{~m}^{3} \mathrm{~m}^{-3}$, are obtained for matric potential values greater than $-10^{-2} \mathrm{~m}$. They decrease to a typical value of $0.03 \mathrm{~m}^{3} \mathrm{~m}^{-3}$ in the central part of the water retention curve and are lower than $0.007 \mathrm{~m}^{3} \mathrm{~m}^{-3}$ for $h<-10$ m. According to the Jurin law, water content near saturation is determined by the proportion of larger pores (up to $1 \mathrm{~mm}$ in diameter) that can be found inside the pozzolan aggregates. Due to the small size of the aggregates (diameter of about $5 \mathrm{~mm}$ ) used in the present study, the proportion of macro 
pores is highly variable from one aggregate to the other, which explains the high variability of the water content near saturation. For lower matric potential values, the water content variability of pozzolan aggregates may be attributed to factors involved in the pozzolan formation. Indeed, pozzolan is a porous siliceous pyroclastic rock, the porosity of which is created by dissolved gas entrapped in lava during scoria emission. The mineralogy of rocks, the proportion of dissolved gas and the temperature of scoria during volcanic eruption, all affect upon the porosity and pore size distribution of pozzolan rocks.

Experimental data are less spread out for the water retention of bark compost (Fig. 3c). Contrary to pozzolan, the water retention measurements for bark compost are obtained on remolded and compacted samples. The porosity of compacted bark compost includes both the matric porosity of individual bark fragments and the structural porosity between bark fragments. As observed for natural soils (Dexter et al., 2008), water retention near saturation is linked to the structural porosity. We observe a very good linear relationship between water retention data and apparent bulk density for matric potentials greater than $-0.7 \mathrm{~m}$ (data not shown). Variability in the water retention measurements is thus very low near saturation, since the apparent bulk density of bark compost samples is accurately controlled. For lower matric potential values, the variability is due to the variability of matric porosity and pore size distribution of bark fragments. This experimental dispersion is relatively low, since the highest standard deviation value is 0.02 $\mathrm{m}^{3} \mathrm{~m}^{-3}$.

Figs. 3a and 3c show that the van Genuchten model adequately fits 
water retention experimental data. Some slight deviations can be found between the mean of the experimental data and the fitted curve for a water potential of about $-1 \mathrm{~m}$. However, these deviations are within the range of the experimental dispersion and they do not question the quality of fit. The estimated uncertainty distributions have a similar behavior to those of the experimental ones: a lower level of uncertainty for compost than for pozzolan, particularly for matric potentials between $-0.01 \mathrm{~m}$ and $-1 \mathrm{~m}$, and a higher level of uncertainty near saturation. For both materials, the most variable and least variable parameter, respectively, are $h_{e}$ and the $n$ exponent, with respective coefficient of variations of approximately $20 \%$ and $1 \%$ for bark compost data, and $35 \%$ and $1 \%$ for pozzolan aggregates data (see Table 1). The $h_{e}$ parameter is a scale parameter, which is related to the entry point of air into the studied material, whereas parameter $n$ is a shape parameter related to the pore size distribution (Kutilek and Nielsen, 1994). A greater scattering of the parameters can thus be linked to the macro porosity variability. A slight variability in exponent $n$ is representative of a homogeneous meso- and micro pore size distribution for both materials.

$K_{\text {Sat }}$ experimental values range from $10^{-7}$ to $10^{-3} \mathrm{~m} \cdot \mathrm{s}^{-1}$ for pozzolan samples (Fig. 3b). The conductivities at saturation of "porous" blocks are higher than those of "dense" blocks (not shown here). The relative standard deviation of $K_{\text {Sat }}$ is quite low for compost (Fig. 3d). This is expected as explained in Appendix A. For the unsaturated part of the hydraulic conductivity curve, the scattering of $(K, h)$ experimental data found for pozzolan and bark compost materials are similar to the scattering of data found for natural soils using the Wind evaporation method (Tamari et al., 1993). We 
observe a lack of experimental values between the data obtained under saturated and unsaturated conditions. This is due to the limited accuracy of matric potential sensors and to the Wind evaporation method: it is necessary to have significant matric potential gradients between two successive sensors to compute an accurate value of the unsaturated hydraulic conductivity. This is usually not achieved close to saturation (Tamari et al., 1993). Despite this limitation, direct computation of $K$ values can be obtained using the Wind evaporation. This is not the case for inverse methods used either in the one-step or the multi-step outflow methods (Hopmans et al., 2002), where only curve parameters are fitted.

Figs. 3b and 3d show that, as expected, the estimated uncertainty of the hydraulic conductivity of pozzolan is greater than that of bark compost. The uncertainty distribution of parameter $n$ is very low for both materials (see Table 2). For the $h_{e}$ and $K_{\text {sat }}$ parameters, the uncertainty is greater for pozzolan material than for bark compost material.

\subsection{Effective hydraulic properties and associated uncertainties}

The uncertainty distributions of the effective water retention and hydraulic conductivity values are presented in Figs. 3e and 3f. Estimated values and standard deviations for the corresponding parameters of the van Genuchten and Mualem-van Genuchten models are displayed in Tables 1 and 2.

The estimated uncertainties for the effective parameters are relatively low and generally lower than those of bark compost and pozzolan under unsaturated conditions. This is mainly due to the size (number of replicates $x$ number of water potential values), and to a lower extent, given this size, to 
the optimal choice of the numerical experimental design used for computing the effective values. These uncertainties increase close to saturation, following the same trend as the one observed for individual components. The standard deviations of most of the van Genuchten and Mualem-van Genuchten parameters estimated for the effective material are lower than the ones estimated for bark compost and pozzolan. The standard deviations of the parameters directly linked to saturated conditions $\left(K_{S a t}\right.$ and $\left.\theta_{s}\right)$ estimated for the effective material, are between those estimated for bark compost and pozzolan.

Various analytical bounds or estimations of effective hydraulic conductivities can be found in the literature (Matheron, 1967; Renard and de Marsily, 1997). The Wiener bounds are the harmonic and arithmetic means of conductivity data and correspond to the effective conductivity of a plane layered porous medium when the water flux is, respectively, perpendicular or parallel to the main orientation of the layered surface. These bounds give a fundamental inequality which is always valid for complex structure patterns (Renard and de Marsily, 1997, Samouëlian et al., 2011). Let $\mu_{a \mid K}$ and $\mu_{h \mid K}$ be the arithmetic and harmonic means of conductivity values, $\omega_{C}$ and $\omega_{P}$ the volumetric proportion of each material, and $K_{C}$ and $K_{P}$ the hydraulic conductivities of compost and pozzolan, respectively. For each water matric potential $h$, the effective conductivity $K_{\text {eff }}$ can be bounded as follows

$$
\mu_{h \mid K}=\frac{1}{\omega_{C} / K_{C}+\omega_{P} / K_{P}} \leq K_{e f f} \leq \mu_{a \mid K}=\omega_{C} K_{C}+\omega_{P} K_{P} .
$$

For a statistically homogeneous and isotropic medium, Matheron (1967) proposes an estimation of the effective conductivity calculated by a geometric 
weighted average of the arithmetic and harmonic means $\mu_{a \mid K}$ and $\mu_{h \mid K}$. This curve is defined by $\mu_{M \mid K}=\mu_{a \mid K}^{\alpha} \mu_{h \mid K}^{1-\alpha}$, where $\alpha=(D-1) / D$ and $D$ is the dimension of the problem.

Figs. 4 show the mean and standard deviation of the 50 effective conductivity values at the 4 water matric potential values, as well as the means of the corresponding Wiener bounds and Matheron estimations. The mean of the effective conductivity values, for each water matric potential value, must be included into the mean of the corresponding Wiener bounds. The crosses which can be observed in Figs. 4 are well-bounded, validating the numerical procedure developed to determine the effective values. Note that the mean of the effective conductivity values is closer to the arithmetic mean curve. It is above the Matheron mean curve before the intersection between the Wiener curves, and below after that intersection. Fig. 5 presents the mean and standard deviation of the effective retention values. It is very close to the arithmetic mean curve. This result is already obtained by several authors (Samouëlian et al., 2007; Samouëlian et al., 2011; Vogel et al., 2008 among others). Under quasi-static conditions and without dynamic effects, the water retention curve presents a capacitive property (Vogel et al., 2008) and the effective water retention curve can be calculated from the additive properties of the local water retention (Samouëlian et al., 2007). The standard deviations of the effective conductivity and retention values computed for the four water potential values are between those obtained for bark compost and pozzolan, and close to the standard deviation of their arithmetic mean (results not shown).

In summary, the methodology using an optimal sampling design leads 
to "well defined" effective properties. We show that the uncertainties of the effective properties are lower than the uncertainties of the properties of each material. The reduction in the level of uncertainty is more pronounced for the van Genuchten parameters associated to the dry part of the properties $\left(\theta_{r}, h_{e}, n\right)$ than for the ones associated to the wet part of the properties $\left(\theta_{s}, K_{\text {sat }}\right)$.

\subsection{Evaluation of the homogenization approach under dynamic evaporation}

Three snapshots of water potential values obtained during the evaporation process are presented in Figs. 6 for the two-component configuration. Heterogeneous spatial distributions of these values are observed at the upper surface of the soil core at $t=2$ days and $t=6$ days. Blue shades represent the gradients due to the geometrical distribution of the two materials. Consequently, isovalues of $h$ do not correspond to horizontal planes. At $t=10$ days, the Dirichlet condition replaces the flux condition at the upper surface, which leads to a uniform spatial distribution of water matric potential values at $z=0$. A gradually stronger vertical gradient could be seen in-depth as evaporation takes place.

Figs. 7 present the uncertainty distributions of water matric potential mean values per depth for the two-component configuration and the corresponding effective homogeneous material configuration. The uncertainty distributions obtained for the effective homogeneous material configuration are clearly less scattered than those obtained for the two-component configuration, and are included in their $[25 t h, 75 t h]$ percentile ranges (not shown here). This is a direct consequence of the lower level of uncertainties of the effective hydraulic properties compared to those of compost and pozzolan. 
Note however that the level of uncertainty obtained here for the simulation of water matric potential might not be representative of the level of uncertainty expected on the simulation of a real evaporation experiment. In this last case, other types of uncertainties, such as uncertainties on initial and boundary conditions for example, must be taken into account.

The mean of these uncertainty distributions for each simulation configuration and depth is also presented in Figs. 7. The curves obtained for the effective homogeneous material and the explicit two-component medium are similar. This confirms the validity of our approach. However, some discrepancies appear as simulation time increases. Table 3 shows the mean water matric potential values and the mean water content values for both configurations at $t_{\max }=10$ days, the final time point of the evaporation simulation. Relative differences between the two configurations are quite high for water matric potentials, and are more pronounced in-depth. However, these relative differences correspond to an area of the core where the porous media are dry. Water content values of both the two-component and the homogeneous material configurations are computed from these water matric potentials using the van Genuchten model and the parameters estimated for the effective material configuration given in Table 1 . These water content values presented in Table 3 show only slight differences between the two configurations, and the relative differences (between $2.1 \%$ and $6.4 \%$ at 10 days) are lower than those of the water matric potentials. Note that these discrepancies may be due to the non-equilibrium flow process, a consequence of the occurrence of transient processes during the dynamic simulation. This point has been showed theoretically by Lewandovska et al. (2004) in the framework of the homoge- 
nization theory : a sink-source term accounting for non-equilibrium processes is added to the Richards equation during the upscaling process. However, this non-equilibirum water flow should be minimized under evaporation scenarios compared to infiltration scenarios because of smoother moisture front (Roth, 2008). Moreover, results obtained by Simunek et al. (2001) show that modeling a non-equilibrium flow modifies the dynamic response for infiltration scenarios, but hardly affects the dynamic response for evaporation scenarios. During the evaporation simulation, the average macroscopic flux is a vertical upward flux. However, the orientation of the local, microscopic water flux could be derived from the vertical direction, due to the presence of local heterogeneous water potential gradients as shown in Figs. 6. The tortuosity of the water flow is, thus, increased. This local tortuosity is a kind of balance between the boundary conditions leading to a vertical macroscopic flux and the contrast of the hydraulic properties of both materials leading to the multidimensional microscopic water flux. The contrast of the hydraulic properties depends on the local water content or matric potentials, and as these variables are time dependent during the dynamic simulation, the tortuosity is also time dependent. This time-dependent tortuosity is not accounted for during the upscaling process since the determination of the effective properties is performed under successive states of equilibrium. Consequently to the time dependency of the local tortuosity, the overall evaporation process of the heterogeneous medium could be accelerated or slowed down compared to the effective material. Therefore, more complex models including nonequilibrium terms might be implemented to study their influence on the dynamic response using the present configuration. 


\section{Conclusion}

The hydraulic properties of a heterogeneous medium are studied at the Darcy scale using two different approaches. The first approach consists in determining the water retention and hydraulic conductivity curves for the components of the heterogeneous medium. The material considered herein is a green roof substrate, which is a composite of compressible materials, bark compost, and of aggregates of volcanic rock, pozzolan. Associated uncertainties are evaluated using a bootstrap method. It is shown, on a virtual evaporation experiment, that the impact of these uncertainties on the simulated water matric potential is high.

The second approach considers the heterogeneous medium as an homogeneous material. Effective water retention and hydraulic conductivity curves are fitted on values computed for several water matric potentials using numerical steady-state scenarios on the heterogeneous material. Associated uncertainties of these properties are also evaluated, considering the uncertainties of the hydraulic properties of each component. As for as we know, this is the first time uncertainty analysis is performed for the computation of effective properties. To that effect, for each hydraulic property, $N$ couples of curves are sampled in the uncertainty distributions of the components in an independent way for a set of water matric potential values. $N$ is set at a high value, and the water matric potential values are optimally chosen. We show that this methodology leads to very low levels of uncertainties in the effective properties of the material. Despite high level of uncertainties on the hydraulic parameters of the two-component material, effective properties of the heterogeneous media can thus be precisely defined using the bootstrap 
method. A direct consequence is that the uncertainties of simulated water potential for the effective material are very low compared to those obtained on the heterogeneous medium. Nevertheless, their mean values are almost similar for dynamic evaporation which confirms the relevance of the approach in these conditions.

Our work shows thus that when non-equilibrium water flow can be neglected, it is preferable to work with the effective parameters rather than with the heterogeneous and distributed parameters in order to reduce uncertainties on the water balance. This is of importance since the uncertainties linked to the experimental setup and to the variability of the materials properties may be high, as shown in the first part of the study.

Simulation models which include a non-equilibrium term could be used in the scope of future studies to evaluate the relevance of effective parameters and the impact of their uncertainties in a larger range of conditions.

\section{Acknowledgment}

This work was supported by the French National Research Agency (ANR) through the "Habitat intelligent et solaire photovoltaique" program (project AGROBAT ANR-09-HABISOL-001). We also thank the CINES (Centre Informatique National de l'Enseignement Supérieur, France) for offering us access to the supercomputer JADE to conduct these calculations successfully. The authors wish to thank Profs. L. Di Pietro, G. Micolau, H. Bolvin and Dr. C. Doussan for their helpful comments and careful readings of the manuscript. 
Atkinson, A.C., Donev, A.N., 1992. Optimum Experimental Designs, volume 8 of Oxford Statistical Science Series, 1st edn. Oxford University Press, Oxford, UK, 352pp.

Bechtold, M., Vanderborght, J., Weihermuller, L., Herbst, M., Gunther, T., Ippisch, O., Kasteel, R., Vereecken, H., 2012. Upward transport in a three-dimensional heterogeneous laboratory soil under evaporation conditions. Vadose Zone J. 11(2).

Besson, A., Cousin, I., Samouëlian, Boizard, H., Richard, G., 2004. Structural heterogeneity of the soil tilled layer as characterized by $2 \mathrm{D}$ electrical resistivity surveying, Soil \& Tillage Res. 79, 239-249.

Bierkens, M.F.P., van der Gaast, J.W.J., 1998. Upscaling hydraulic conductivity: theory and examples from geohydrological studies. Nutri. Cycl. Agroecosyst. 50, 193-207.

Brunner, P., Doherty, J., Simmons, C.T., 2012. Uncertainty assessment and implications for data acquisition in support of integrated hydrologic models. Water Resour. Res. 48(7).

Carter, T., Jackson, C.R., 2007. Vegetated roofs for stormwater management at multiple spatial scales. Landsc. Urban Plan. 80, 84-94.

Celia, M.A., Bouloutas, E.T., Zarba, R.L., 1990. A general massconservative numerical solution for the unsaturated flow equation. Water Resour. Res. 26(7), 1483-1496.

Chossat, J.C., 2005. La mesure de la conductivité hydraulique dans les sols 
- Choix des méthodes, 1st edn. Tec et Doc - Lavoisier Editions, London, Paris, New York, 720pp.

Christiaens, K., Feyen, J., 2001. Analysis of uncertainties associated with different methods to determine soil hydraulic properties and their propagation in the distributed hydrological MIKE SHE model. J. Hydrol. 246(1-4), 63-81.

Coleman, T.F., Li, Y., 1996. An Interior, Trust Region Approach for Nonlinear Minimization Subject to Bounds. SIAM J. Optimiz. 6(2), 418-445.

Coppola, A., Basile, A., Comegna, A., Lamaddalena, N., 2009. Monte Carlo analysis of field water flow comparing uni- and bimodal effective hydraulic parameters for structured soil. J. Contam. Hydrol. 104(1-4), 153-165.

Dane, J.H., Hopmans, J.W., 2002. Methods of soil analysis: Part 4 Physical methods, In: Dane, J.H., Topp, G.C. and Campbell, G.S. (Ed.) Soil Science Society of America, Madison:SSSA book series 5, 1662pp.

Danquigny, C., Ackerer, P., 2005. Experimental determination of equivalent parameters of a heterogeneous porous medium under uniform or radial flow. C. R. Geoscience. 337(6), 563-570.

Dexter, A.R., Czyz, E.A., Richard, G., Reszkowska, A., 2008. A userfriendly water retention function that takes account of the textural and structural pore spaces in soil. Geoderma. 143(3-4), 243-253.

Dhatt, G., Touzot, G., Lefrançois, E., 2007. Méthode des éléments finis, 4th edn. Hermes Science Publications - Lavoisier Editions, London, Paris, New York, 601pp. 
Duliu, O.G., 1999. Computer axial tomography in geosciences : an overview.Earth-Sci. Rev. 48, 265-281.

Efron, B., Tibshirani, R., 1994. An Introduction to the Bootstrap, Monographs on Statistics \& Applied Probability (Book 57), 1st edn. Chapman \& Hall/CRC, London, UK, 456pp.

Fityus, S.G., Smith, D.W., 2001. Solution of the unsaturated soil moisture equation using repeated transforms. Int. J. Numer. Anal. Methods Geomech. 25(15), 1501-1524.

Forschungsgesellschaft Landschaftsentwicklung Landschaftsbau e.V.-FLL, 2002. Guidelines for the planning, execution and upkeeping of green-roof sites, 1st edn. Forschungsgesellschaft Landschaftsentwicklung Landschaftsbau e. V. - FLL, Bonn, Germany, 97 pp. URL: http://www.fll.de

Hardelauf, H., Javaux, M., Herbst, M., Gottschalk, S., Kasteel, R., Vanderborght, J., Vereecken, H., 2007. PARSWMS: A parallelized model for simulating three-dimensional water flow and solute transport in variably saturated soils. Vadose Zone J. 6(2), 255-259.

Herbst, M., Gottschalk, S., Reissel, M., Hardelauf, H., Kasteel, R., Javaux, M., Vanderborght, J., Vereecken, H., 2008. On preconditioning for a parallel solution of the Richards equation. Comput. \& Geosci. 34(12), 1958-1963.

Hopmans, J.W., Šimůnek, J., Romane, N., Durner, W., 2002. Methods of Soil analysis - Part 4 Physical Methods, In: Dane, J.H., Topp, G.C. and Campbell, G.S. (Ed.) Soil Science Society of America, Madison:SSSA book series 5, 1662pp. 
Huang, K., Mohanty, B.P., van Genuchten, Mth., 1996. A new convergence criterion for the modified Picard iteration method to solve the variably saturated flow equation. J. Hydrol. 178(1-4), 69-91.

Javaux, M., Vanclooster, M., 2006a. Three-dimensional structure characterisation and transient flow modelling of a variably saturated heterogeneous monolith. J. Hydrol. 327(3-4), 516-524.

Javaux, M., M. Vanclooster, M., 2006b. Scale-dependency of the hydraulic properties of a variably saturated heterogeneous sandy subsoil. J. Hydrol. 327, 376-388.

Ju, S.H., Kung, K.J.S., 1997. Mass types, element orders and solution schemes for the Richards equation. Comput. \& Geosci. 23(2) 175-187.

Kavetski, D., Binning, P., Sloan, S.W., 2001. Adaptive time stepping and error control in a mass conservative numerical solution of the mixed form of Richards equation. Adv. Water Resour. 24(6), 595-605.

Khaleel, R., Yeh, T.C.J., Lu, Z., 2002. Upscaled flow and transport properties for heterogeneous unsaturated media, Water Resour. Res. 38, 1053.

Kutilek, M., Nielsen, D.R., 1994. Soil Hydrology, 1st edn. Catena Verlag GMBH, Reiskirchen, Germany, 370pp.

Lewandowska, J., Szymkiewicz, A., Burzynski, K., Vauclin, M., 2004. Modeling of unsaturated water flow in double-porosity soils by the homogenization approach. Adv. Water Resour. 27, 283-296. 
Ma, D., Shao, M., Zhang, J., Wang, Q., 2010. Validation of an analytical method for determining soil hydraulic properties of stony soils using experimental data. Geoderma. 159, 262-269.

Manly, B.F.J., 2006. Randomization, Bootstrap and Monte Carlo Methods in Biology, 3rd edn. Chapman \& Hall/CRC, London, UK, 480pp.

Mantaglou, A., Gelhar, L.W., 1987. Stochastic modeling of large-scale transient unsaturated flow flow systems. Water Resour. Res. 23, 37-46.

Matheron G., 1967. Eléments pour une Théorie des Milieux Poreux, 1st edn. Masson et Cie, Paris, France, 168pp.

Mohrath, D., Bruckler, L., Bertuzzi, P., Gaudu, J.C., Bourlet, M., 1997. Error analysis of an evaporation method for determining hydrodynamic properties in unsaturated soil. Soil Sci. Soc. Am. J. 61(3), 725-735.

Monnier, G., Stengel, P., Fiès, J.C., 1973. Une méthode de mesure de la densité apparente de petits agglomérats terreux. Application à l'analyse des systèmes de porosité du sol. Ann. agron. 24(5), pp. 533-545.

Ouldboukhitine, S.E., Belarbi, R., Djedjig, R., 2012. Characterization of green roof components: Measurements of thermal and hydrological properties. Build Environ. 56, 78-85.

Pan, F., Ye, M., Zhu, J., Wu, Y.S., Hu, B.X., Yu, Z., 2009. Numerical evaluation of uncertainty in water retention parameters and effect on predictive uncertainty. Vadose Zone J. 8(1), 158-166. 
Peters, A., Durner, W., 2008. Simplified evaporation method for determining soil hydraulic properties. J. Hydrol. 356(1-2), 147-162.

Renard, Ph., de Marsily, G., 1997. Calculating equivalent permeability: a review. Adv. Water Resour. 20(5-6), 253-278.

Renaud, J., Cloke, H., Wang, Y., Anderson, M., Wilkinson, P., Lloyd, D., 2003. Simulation numérique d'écoulements en milieu poreux avec l'équation de Richards. Eur. J. Comput. Mech. 12(2-3), 203-220.

Roth, K., 2008. Scaling of water flow through porous media and soils. Eur. J. of Soil Sci. 59, 125-130.

Russo, D., 1992. Upscaling of hydraulic conductivity in partially saturated heterogeneous porous formation. Water Resour. Res. 28, 397-409.

Samouëlian, A., Vogel, H.J., Ippisch, O., 2007. Upscaling hydraulic conductivity based on the topology of the sub-scale structure. Adv. Water Resour. 30(5), 1179-1189.

Samouëlian, A., Cousin, I., Dagès, C., Frison, A., Richard, G., 2011. Determining the effective hydraulic properties of a highly heterogeneous soil horizon. Vadose Zone J. 10(1), 450-458.

Simunek J., Wendroth, O., Wypler, N., van Genuchten, M.Th., 2001. Nonequilibrium water flow characterized by means of upward infiltration experiments. Eur. J. Soil. Sci. 52(1), 13-24.

Tabbagh, A., Dabas, M., Hesse, A., Panissod, C., 2000. Soil resistivity: a non-invasive tool to map soil structure horizonation. Geoderma 97, 393-404. 
Tamari, S., Bruckler, L., Halbertsma, J., Chadoeuf, J., 1993. A simple method for determining soil hydraulic properties in the laboratory. Soil Sci. Soc. Am. J. 57(3), 642-651.

Tracy, F.T., 2007. Three-dimensional analytical solutions of Richards' equation for a box-shaped soil sample with piecewise-constant head boundary conditions on the top. J. Hydrol. 336(3-4), 391-400.

Vanderborght, J., Kasteel, R., Herbst, M., Javaux, M., Thiéry, D., Vanclooster, M., Mouvet, C., Vereecken, H., 2005. A set of analytical benchmarks to test numerical models of flow and transport in soils. Vadose Zone J. 4(1), 206-221.

van Genuchten, MTh., 1980. A closed-form equation for predicting the hydraulic conductivity of unsaturated soils. Soil Sci. Soc. Am. J. 44(5), 892898.

Vereecken, H., Kasteel, R., Vanderborght, J., Harter, T., 2007. Upscaling hydraulic properties and soil water flow processes in heterogeneous soils: a review. Vadose Zone J. 6, 1-28.

Vogel, H.J., Roth, K., 1998. A new approach for determining soil hydraulic functions. Eur. J. Soil Sci. 49(4), 547-556.

Vogel, H.J., Samouëlian, A., Ippisch, O., 2008. Multi-step and two-step experiments in heterogeneous porous media to evaluate the relevance of dynamic effectes. Adv. Water Resour. 31(1), 181-188.

Vogel, H.J., Weller, U., Ippisch, O., 2010. Non-equilibrium in soil hydraulic modelling. J. Hydrol. 393(1-2), 20-28. 
700 Wildenschild, D., Jensen, K.H., 1999a. Laboratory investigations of effective 701 flow behavior in unsaturated heterogeneous sands. Water Resour. Res. 35, $702 \quad 17-27$.

${ }_{703}$ Wildenschild, D., Jensen, K.H., 1999b. Numerical modeling of observed 704 effective flow behavior in unsaturated heterogeneous sands. Water Resour. 705 Res. 35, 29-42.

706 Yang, J., Yu, Q., Gong, P., 2008. Quantifying air pollution removal by 707 greenroofs in chicago. Atmos. Environ. 42, 7266-7273.

Yeah, T.C.J., Gelhar, L.W., Gutjahr, A.L., 1985. Stochastic analysis of unsaturated flow in heterogeneous soils. 1. Statistically isotropic media. Water Resour. Res. 21, 447-456.

Zhang, Y., Gable, C.W., Sheets, B., 2010. Equivalent hydraulic conductivity of three-dimensional heterogeneous porous media: An upscaling study based on an experimental stratigraphy. J. Hydrol. 388, 304-320. 


\begin{tabular}{|c|c|c|c|c|c|}
\hline & & $\begin{array}{l}\theta_{r} \\
\mathrm{~m}^{3} \mathrm{~m}^{-3}\end{array}$ & $\begin{array}{l}\theta_{s} \\
\mathrm{~m}^{3} \mathrm{~m}^{-3}\end{array}$ & $\begin{array}{l}\left|h_{e}\right| \\
\mathrm{m}\end{array}$ & $n$ \\
\hline \multirow[t]{2}{*}{ Compost } & $\begin{array}{l}\text { Estimat. } \\
\text { value }\end{array}$ & $8.66 e^{-2}$ & $5.55 e^{-1}$ & $7.47 e^{-3}$ & 1.22 \\
\hline & $\sigma$ & $\begin{array}{l}5.92 e^{-3} \\
(6.9 \%)\end{array}$ & $\begin{array}{l}1.27 e^{-2} \\
(2.3 \%)\end{array}$ & $\begin{array}{l}1.63 e^{-3} \\
(21.8 \%)\end{array}$ & $\begin{array}{l}1.01 e^{-2} \\
(0.8 \%)\end{array}$ \\
\hline \multirow[t]{2}{*}{ Pozzolan } & $\begin{array}{l}\text { Estimat. } \\
\text { value }\end{array}$ & $0($ fixed $)$ & $3.53 e^{-1}$ & $5.88 e^{-3}$ & 1.29 \\
\hline & $\sigma$ & $0($ fixed $)$ & $\begin{array}{l}1.63 e^{-2} \\
(4.6 \%)\end{array}$ & $\begin{array}{l}2.05 e^{-3} \\
(34.9 \%)\end{array}$ & $\begin{array}{l}1.11 e^{-2} \\
(0.9 \%)\end{array}$ \\
\hline \multirow[t]{2}{*}{$\begin{array}{l}\text { Effective } \\
\text { material }\end{array}$} & $\begin{array}{l}\text { Estimat. } \\
\text { value }\end{array}$ & $3.81 e^{-2}$ & $4.35 e^{-1}$ & $6.71 e^{-3}$ & 1.26 \\
\hline & $\sigma$ & $\begin{array}{l}7.17 e^{-4} \\
(1.9 \%)\end{array}$ & $\begin{array}{l}1.36 e^{-2} \\
(3.1 \%)\end{array}$ & $\begin{array}{l}1.04 e^{-3} \\
(15.5 \%)\end{array}$ & $\begin{array}{l}2.74 e^{-3} \\
(0.2 \%)\end{array}$ \\
\hline
\end{tabular}

Table 1: Estimated values and associated uncertainties of the van Genuchten model parameters for water retention of compost, pozzolan and effective material. 


\begin{tabular}{|c|c|c|c|c|}
\hline & & $\begin{array}{l}K_{\text {Sat }} \\
\mathrm{m} . \mathrm{s}^{-1}\end{array}$ & $\begin{array}{l}\left|h_{e}\right| \\
\mathrm{m}\end{array}$ & $n$ \\
\hline \multirow[t]{2}{*}{ Compost } & $\begin{array}{l}\text { Estimat. } \\
\text { value }\end{array}$ & $3.04 e^{-3}$ & $9.72 e^{-3}$ & 1.12 \\
\hline & $\sigma$ & $\begin{array}{l}1.03 e^{-4} \\
(3.4 \%)\end{array}$ & $\begin{array}{l}2.79 e^{-4} \\
(2.9 \%)\end{array}$ & $\begin{array}{l}8.87 e^{-3} \\
(0.8 \%)\end{array}$ \\
\hline \multirow[t]{2}{*}{ Pozzolan } & $\begin{array}{l}\text { Estimat. } \\
\text { value }\end{array}$ & $6.94 e^{-6}$ & $6.01 e^{-1}$ & 1.02 \\
\hline & $\sigma$ & $\begin{array}{l}7.95 e^{-6} \\
(114.6 \%)\end{array}$ & $\begin{array}{l}9.90 e^{-1} \\
(164.7 \%)\end{array}$ & $\begin{array}{c}9.64 e^{-3} \\
(0.9 \%)\end{array}$ \\
\hline \multirow[t]{2}{*}{$\begin{array}{l}\text { Effective } \\
\text { material }\end{array}$} & $\begin{array}{l}\text { Estimat. } \\
\text { value }\end{array}$ & $9.36 e^{-4}$ & $2.28 e^{-2}$ & 1.05 \\
\hline & $\sigma$ & $\begin{array}{l}8.91 e^{-5} \\
(9.5 \%)\end{array}$ & $\begin{array}{l}8.01 e^{-4} \\
(3.5 \%)\end{array}$ & $\begin{array}{l}1.91 e^{-3} \\
(0.2 \%)\end{array}$ \\
\hline
\end{tabular}

Table 2: Estimated values and associated uncertainties of the Mualem-van Genuchten model parameters for hydraulic conductivity of compost, pozzolan and effective material. 


\begin{tabular}{|c|c|c|c|c|}
\hline & & $\begin{array}{l}\text { Depth }= \\
-0.2 \mathrm{~cm}\end{array}$ & $\begin{array}{l}\text { Depth }= \\
-1.1 \mathrm{~cm}\end{array}$ & $\begin{array}{l}\text { Depth }= \\
-4.8 \mathrm{~cm}\end{array}$ \\
\hline \multirow[t]{3}{*}{$\begin{array}{l}\text { Water matric } \\
\text { potential means } \\
(\mathrm{m})\end{array}$} & $\begin{array}{l}\text { Effective homo- } \\
\text { geneous material } \\
\text { configuration }\end{array}$ & -45.78 & -15.33 & -5.68 \\
\hline & $\begin{array}{l}\text { Two-component con- } \\
\text { figuration }\end{array}$ & -53.71 & -21.47 & -8.36 \\
\hline & Relative differences & $14.8 \%$ & $28.6 \%$ & $32.1 \%$ \\
\hline \multirow[t]{3}{*}{$\begin{array}{l}\text { Water content } \\
\text { means }\left(\mathrm{m} \cdot \mathrm{m}^{-3}\right)\end{array}$} & $\begin{array}{l}\text { Effective homo- } \\
\text { geneous material } \\
\text { configuration }\end{array}$ & $7.81 \mathrm{e}-2$ & $9.12 \mathrm{e}-2$ & 10.69 e-2 \\
\hline & $\begin{array}{l}\text { Two-component con- } \\
\text { figuration }\end{array}$ & $7.65 \mathrm{e}-2$ & 8.68 e-2 & $10.00 \mathrm{e}-2$ \\
\hline & Relative differences & $2.1 \%$ & $4.9 \%$ & $6.4 \%$ \\
\hline
\end{tabular}

Table 3: Means of all water matric potential and water content values for several depth and for the two-component and effective material configurations. 


\section{Appendix A. Experimental procedures}

The green roof substrate under study is composed of a combination of $40 \%$ organic material (bark compost) and 60\% volcanic material (pozzolan). This combination corresponds to the volumetric proportions which are actually used in in situ environments.

Determination of solid density and apparent bulk density of compost and pozzolan

Pozzolan materials are extracted in a quarry located in the "Massif Central" mountain, in the center of France. Chemical composition of pozzolan is given by the operator of the quarry $\left(\mathrm{SiO}_{2}: 42-55 \% ; \mathrm{Al}_{2} \mathrm{O}_{3}: 12-24 \% ; \mathrm{Fe}_{2} \mathrm{O}_{3}\right.$ : $8-20 \%$ ). The pozzolan aggregate size distribution is the following: $2 / 3$ of the pozzolan aggregate diameters ranged from 3 to $6 \mathrm{~mm}$, and $1 / 3$ from 7 to $15 \mathrm{~mm}$. Bark compost is provided by an industrial partner and the precise composition of this compost is confidential.

The properties of the two materials are measured on samples of both materials, and are measured at the same bulk density $\left[M L^{-3}\right]$ as they occur in the actual substrate.

Samples are crunched, sieved at $315 \mu \mathrm{m}$ and air dried in the oven for $24 \mathrm{~h}$ at $105^{\circ} \mathrm{C}$. Solid particles are then placed in the measurement chamber of a $\mathrm{He}$ pycnometer and solid bulk density is determined using the Boyle law (Dane and Hopmans, 2002). The apparent bulk density of pozzolan aggregates is measured on replicates with diameters varying from 7 to $15 \mathrm{~mm}$, using the Archimede law and buoyancy measurements in water. The aggregates are previously saturated in water for $24 \mathrm{~h}$ (Monnier et al., 1973). The apparent 
bulk density of the composite substrate is measured using the core method (Dane and Hopmans, 2002) adapted for compacted and remolded samples. Since bark compost is a compressible material, contrary to pozzolan, we follow the procedure described hereafter to be sure that the density of pure bark compost samples is the same as the density of bark compost present in the actual composite substrate: the complex substrate is characterized following a standard compaction methodology, namely the Proctor compaction test, standard DIN 18127. The sample is struck 6 times by a $4.5 \mathrm{~kg}$ Proctor hammer from a height of $45 \mathrm{~cm}$ to obtain adequate compaction. The compacted sample obtained following this methodology is supposed to be representative of the in situ industrial execution of the green roofs (Forschungsgesellschaft Landschaftsentwicklung Landschaftsbau e. V. - FLL, 2002). By knowing, on the one hand, the apparent bulk density of the substrate and of the pozzolan aggregates and, on the other hand, the solid density of bark compost and of pozzolan, we extrapolate the apparent bulk density of bark compost in the actual composite substrate.

All the measured properties are displayed in Table A.4.

\section{Determination of water retention and associated uncertainties}

Water retention measurements are obtained using the suction table for small suctions $(0.05,0.54,1.03,2.01,3.97,6.91 \mathrm{kPa})$ and the pressure plate extractors for large suctions $(10,30,50,100,300,500$ and $1500 \mathrm{kPa}$ ) (Dane and Hopmans, 2002). The total number of replicates differs for pozzolan and bark compost materials.

1. Since pozzolan aggregates are a natural material with a high level of variability due to geological variations occurring during their formation, 


\begin{tabular}{|c|c|c|c|c|}
\hline & & $\begin{array}{l}\text { Pozzolan ag- } \\
\text { gregate }\end{array}$ & $\begin{array}{l}\text { Complex } \\
\text { substrate }\end{array}$ & $\begin{array}{ll}\text { Bark com- } & \text { post }\end{array}$ \\
\hline \multirow{2}{*}{$\begin{array}{l}\text { Particle } \\
\text { density } \\
\left(\mathrm{kg} \cdot \mathrm{m}^{-3}\right)\end{array}$} & Mean & 3.000 & - & 1.670 \\
\hline & $\begin{array}{l}\sigma \\
\text { coefficient of } \\
\text { variation }\end{array}$ & $\begin{array}{l}0.020 \\
0.7 \%\end{array}$ & - & $\begin{array}{l}0.040 \\
2.4 \%\end{array}$ \\
\hline \multirow{2}{*}{$\begin{array}{l}\text { Apparent } \\
\text { bulk density } \\
\left(\mathrm{kg} \cdot \mathrm{m}^{-3}\right)\end{array}$} & Mean & 1.520 & 0.822 & 0.195 \\
\hline & $\begin{array}{l}\sigma \\
\text { coefficient of } \\
\text { variation }\end{array}$ & $\begin{array}{l}0.230 \\
15.1 \%\end{array}$ & $\begin{array}{l}0.002 \\
0.2 \%\end{array}$ & $\begin{array}{l}0.003 \\
1.5 \%\end{array}$ \\
\hline
\end{tabular}

Table A.4: Mean and standard deviation values of the particle and apparent bulk densities for bark compost, pozzolan aggregate and complex substrate. 
a large number of replicates are required to counter the effects of this natural heterogeneity. 10 different aggregates are used for each suction point. The aggregates taken for apparent bulk density measurements are also used for the $0 \mathrm{kPa}$ suction point. Water saturated aggregates are gently placed onto a fine layer of kaolinite paste to ensure a good contact between aggregate pores and the sand layer (for suction tables) or the porous plate (for pressure plate extractors). A set time for sample equilibration of 3 days is respected.

2. Bark compost samples are compacted in the laboratory following a standard procedure to obtain a predetermined density of compost into the composite substrate, the variability of which is very low (see Table A.4). A low number of replicates are, therefore, required. 5 different bark compost samples are used for each suction point. To account for any natural variability in the density of bark compost within the composite substrate, samples are compacted in small cylinders ranging from 0.184 and 0.213 g.cm ${ }^{-3}$. Mean bulk density is $0.200 \mathrm{~g} . \mathrm{cm}^{-3}$, which differs slightly from the theoretical bulk density of compost within the composite substrate $\left(0.195\right.$ g.cm $\left.{ }^{-3}\right)$, due probably to experimental approximations. After initial saturation, samples are placed onto the suction table or porous plate. Kaolinite paste is also used to increase the quality of the capillary connectivity between the sample and the sand layer or porous plate. The time needed for sample equilibrium is over one week for each suction point and is controlled by monitoring the water flow out of the pressure chamber. 


\section{Determination of hydraulic conductivity and associated uncertainties}

Hydraulic conductivity is measured for pozzolan and bark compost under water-saturated and unsaturated conditions using two different methods. Hydraulic conductivity at saturation $K_{\text {Sat }}\left[L T^{-1}\right]$ is measured using a constant head permeameter (Chossat, 2005) for pozzolan cores and bark compost samples. Unsaturated hydraulic conductivity is measured using the Wind evaporation method (Tamari et al., 1993). Samples used for determining the hydraulic conductivity at saturation are also used to determine the unsaturated hydraulic conductivities. As is the case of the water retention curve, the number of replicates differs for pozzolan and bark compost, and is adapted to the different variability of both materials: 10 replicates are used for pozzolan and 1 replicate is used for bark compost.

Samples of pozzolan are extracted from large blocks (volume of approximately $\left.1 \mathrm{dm}^{-3}\right)$. A classification of these blocks is initially made based on the visible porosity and the estimated bulk density: an equal number of "compact" and "porous" blocks in equal quantities are identified. 10 replicates are then cored from both types of blocks. The vertical walls of the clods are surrounded by heat shrink tubing to avoid preferential water flow along the walls during measurements. The uncertainty distribution of the measured hydraulic conductivity at saturation used in section 3.1 is estimated as the experimental variability of $K_{\text {Sat }}$ measurements.

Samples of bark compost are obtained after their compaction up to 0.195 g. $\mathrm{cm}^{-3}$ in cylinders. A single replicate is used, since a low experimental variability is expected as the compaction procedure is accurately performed and leads to a very low level of variability in the porosity obtained (see 
Table A.4). Porosity $\phi$ of bark compost is measured for this single sample. The uncertainty of the measured saturated hydraulic conductivity could thus be estimated from the variability in the porosity values using the KozenyCarman model (Kutilek and Nielsen, 1994). A simple error propagation computed using this model, assuming a log-normal distribution of $K_{\text {Sat }}$, gives rise to

$$
\operatorname{var}\left[\log \left(K_{\text {Sat }}\right)\right]=\left(\frac{3-\phi}{\phi(1-\phi)}\right)^{2} \operatorname{var}(\phi) .
$$

For measuring the unsaturated hydraulic conductivity, pozzolan clods and bark compost are equipped with sufficient microtensiometers to obtain an accurate estimation of their hydraulic properties (Tamari et al., 1993). After the sample saturation, water is allowed to evaporate from the upper surface of each core under laboratory conditions, i.e. conditioned atmosphere at 24 ${ }^{\circ} \mathrm{C}$ under atmospheric pressure. The base of each core is sealed to prevent downward flux.

\section{Appendix B. C ++ parallelized code}

The three-dimensional variably saturated flow modeling is based on the non-linear Richards equation. The mixed form of Richards equation obtained by combining the mass conservation law with the generalized Darcy equation is

$$
\frac{\partial \theta(h, \mathbf{x}, t)}{\partial t}=\nabla \cdot(K(h, \mathbf{x}, t) \nabla(h(\mathbf{x}, t)+z))
$$

where $z$ is the upward vertical coordinate. Constitutive functions depending on the materials considered link $h(\mathbf{x}, t), \theta(h, \mathbf{x}, t)$ and $K(h, \mathbf{x}, t)$ and close (B.1). Various initial and boundary conditions can complete the parabolic system of partial differential equations. 
Water flow is solved using the mixed form of the Richards equation, for which a perfect mass balance is ensured (Celia et al., 1990; Kavetski et al., 2001; Renaud et al., 2003 to cite a few). Spatial discretization is performed using the Galerkin-type linear isoparametric finite elements (Dhatt et al., 1984; Ju and Kung, 1997). The modified Picard iteration scheme is implemented in a fully implicit Euler time discretization. Different convergence criterion can be used, as explained in Huang et al. (1996). An adaptive time step adjustment is implemented to improve numerical efficiency (Kavetski et al., 2001). Time and spatial discretizations result in the following system of linear equations

$$
\begin{aligned}
& {\left[\frac{\left[M^{t+1, m}\right]\left[C^{t+1, m}\right]}{\Delta t}+\left[K^{t+1, m}\right]\right]\left\{\delta_{u}^{m}\right\}=} \\
& \left\{F^{t+1, m}\right\}-\frac{\left[M^{t+1, m}\right]}{\Delta t}\left\{\theta^{t+1, m}\right\}+\frac{\left[M^{t+1, m}\right]}{\Delta t}\left\{\theta^{t}\right\}-\left[K^{t+1, m}\right]\left\{u^{t+1, m}(\mathrm{~B} .2)\right.
\end{aligned}
$$

where $t$ and $m$ denote, respectively, time and inner iteration levels, $u=h+z$ and $\delta_{u}^{m}=u^{t+1, m+1}-u^{t+1, m},[M]$ and $[K]$ are the global mass and stiffness matrices, $F$ includes the source/sink terms and $[C(h, \mathbf{x}, t)]$ is the specific moisture capacity function matrix.

A $\mathrm{C}++$ object oriented code (FAFEMO) has been developed to solve (B.2) and to determine the water matric potential and the water flux at each node/boundary of the finite element mesh grid for an unsaturated medium. Our numerical results are found to corroborate analytical results published by Tracy (2007), Fityus and Smith (2001) or purely numerical results proposed by Vanderborght et al. (2005).

Due to the specific composition of the green roof substrate, very fine spatial grids are required, leading to huge CPU time and storage cost. This 
846 major drawback is overcome by a parallelization of code FAFEMO with 847 a similar parallelization strategy as the one presented in Hardelauf et al., 848 2007, or Herbst et al., 2008. MPI (http://www.mpi-forum.org/) and PETSc 849 (http://www.mcs.anl.gov/petsc/) libraries were used herein to parallelize the ${ }_{850} \mathrm{C}++$ sequential code. 
LaTeX Source Files
Click here to download LaTeX Source Files: JH_V27_unmarked.tex

LaTeX Source Files
Click here to download LaTeX Source Files: JH_V27_unmarked.tex

Click here to download LaTeX Source Files: JH_V27_unmarked.tex

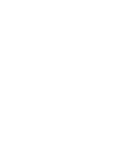

$\sqrt{2}$

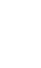

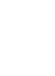
(1) (1) (1) (1) (1) (1) (1)

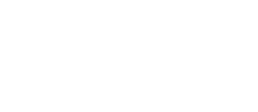

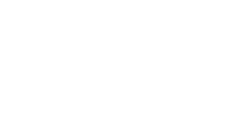

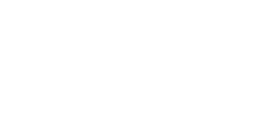

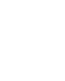

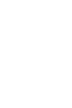

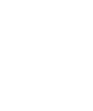

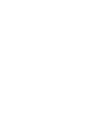

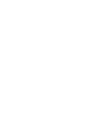

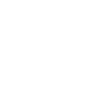

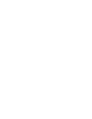

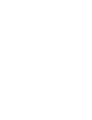

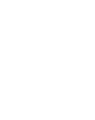

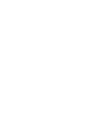
-

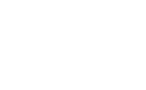
$x^{2}+x^{2}$ (n) $\sqrt{2+2}+x^{2}$

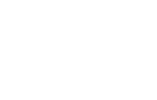

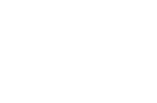

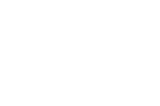

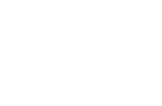


Highlights:

- The effective hydraulic properties of real heterogeneous media were evaluated at the Darcy scale.

- The suitability of the homogenization approach was shown in a case of dynamic evaporation simulations.

- We accounted for the uncertainties for the hydraulic properties of each material.

- A reduction in the level of uncertainties was obtained for the effective hydraulic properties. 


\section{List of captions}

Figure 1:

General flowchart of the approach. \# is used to symbolise several samples or values. Blue color is used for the description of the processing of the heterogeneous material and its individual components, green color for the effective material, red color for the treatment of uncertainties.

Figure 2:

Typical heterogeneous mesh for the two-component configuration. Black or white zones correspond, respectively, to the pozzolan or bark compost components.

Figure 3:

Uncertainty distributions of a) pozzolan, c) compost and e) effective water retention values. Crosses and error bars represent means and \pm 1 standard deviations of measured or simulated data and curves represent $0.5,2.5,25,75,97.5,99.5$ percentiles and median of the estimated water retention uncertainty distributions. Vertical axes are in log scale.

Uncertainty distributions of b) pozzolan, d) compost and f) effective hydraulic conductivity values. Data obtained for pozzolan samples are represented with the same symbol since experimental scattering largely overlaps from one sample to the other. Vertical and horizontal axes are in log scale. Water potential axes are cut to artificially plot asymptotic behaviour and measured values at $0 \mathrm{~m}$ in $\log$ scale.

Figure 4:

Effective conductivity values computed from 200 compost and pozzolan properties for 4 water matric potential values. Crosses represent the mean and \pm 1 standard deviations of the 50 computed values per water matric potential point, dotted lines are the means of the Wiener bounds and the plain line is the Matheron curve obtained from these mean curves. Fig. b) is an enhanced view of Fig. a). Vertical and horizontal axes are in log scale.

Figure 5:

Effective retention values computed from 200 compost and pozzolan properties for 4 water matric potential values. Crosses represent the mean and \pm 1 standard deviations of the 50 computed values per water matric potential 
point and the plain line is the mean of the arithmetic mean curves obtained from the compost and pozzolan retention curves used. The vertical axis is in $\log$ scale.

Figure 6:

Snapshots of the evolution of the water potential according to time, at a) $t=2$ days, b) $t=6$ days and c) $t=10$ days. Scales for water matric potential $(\mathrm{m})$ are different for each snapshot.

\section{Figure 7:}

Uncertainty distributions of water matric potential values obtained by simulating evaporation using the heterogeneous green roof substrate (black curves) and using the corresponding computed effective homogeneous material (red curves). Dotted or solid curves represent, respectively, 0.5, 99.5 percentile or median values for the water matric potential distributions. The 4 graphs correspond to 4 various depths of the core. 


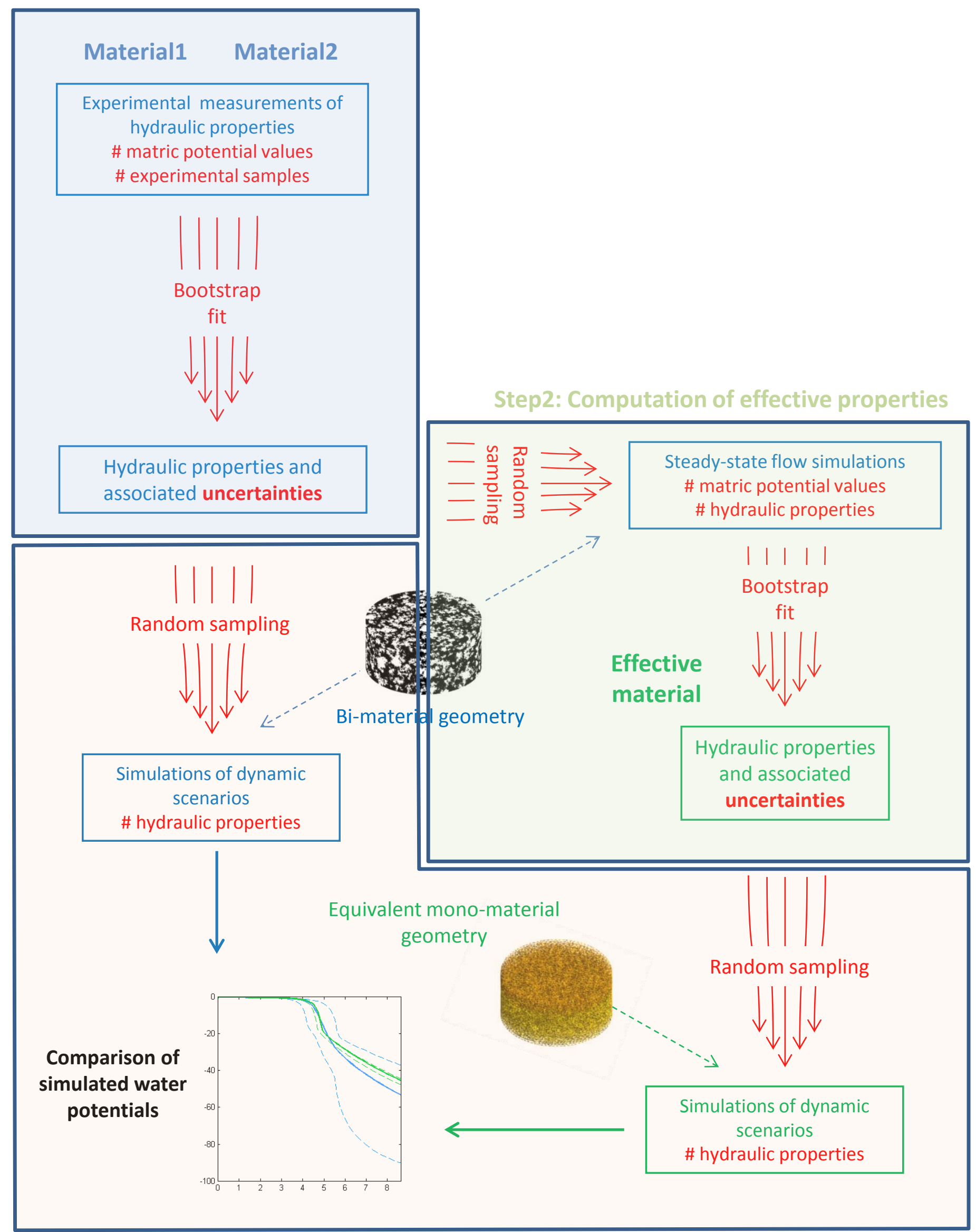




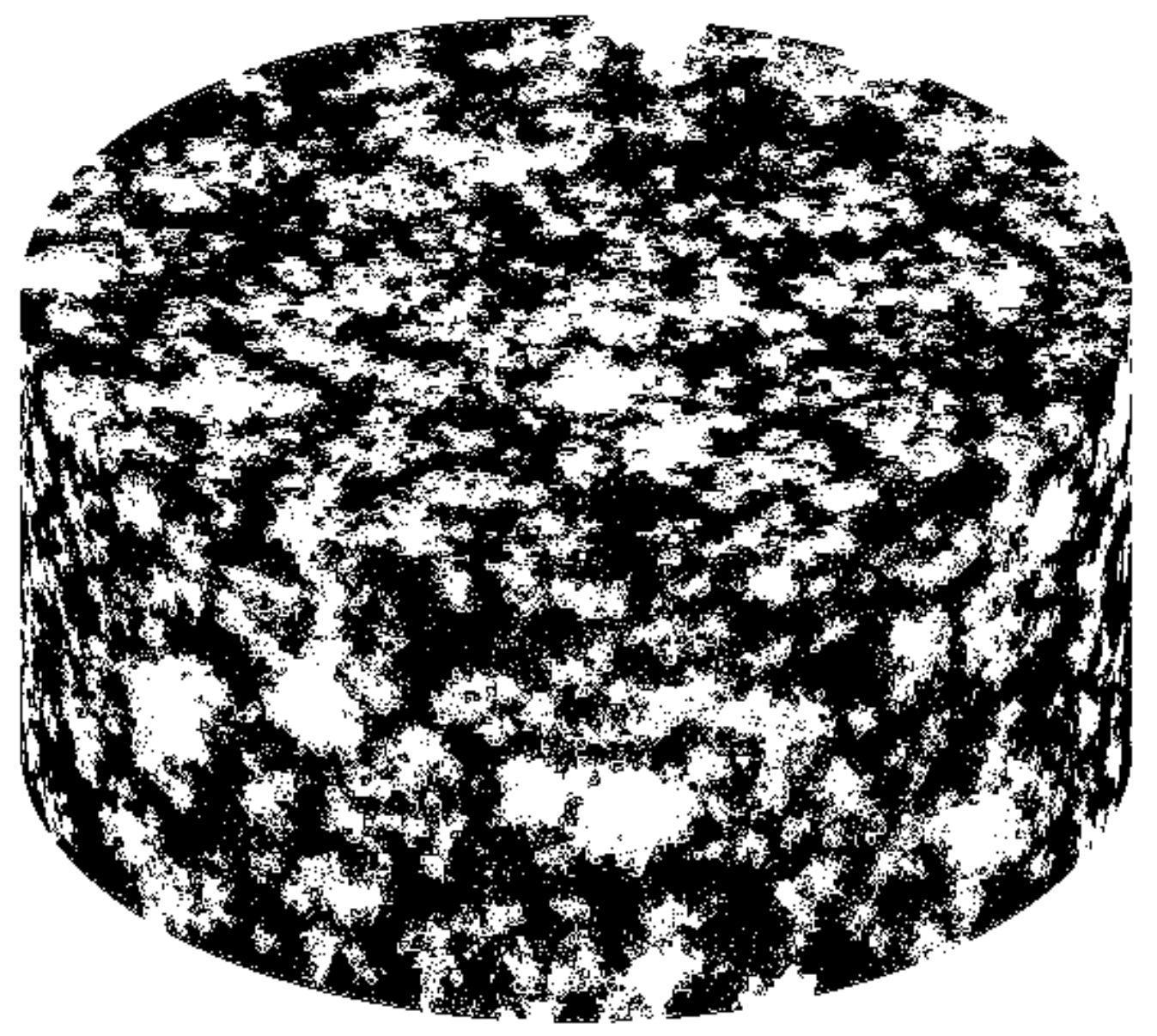


Figure 3

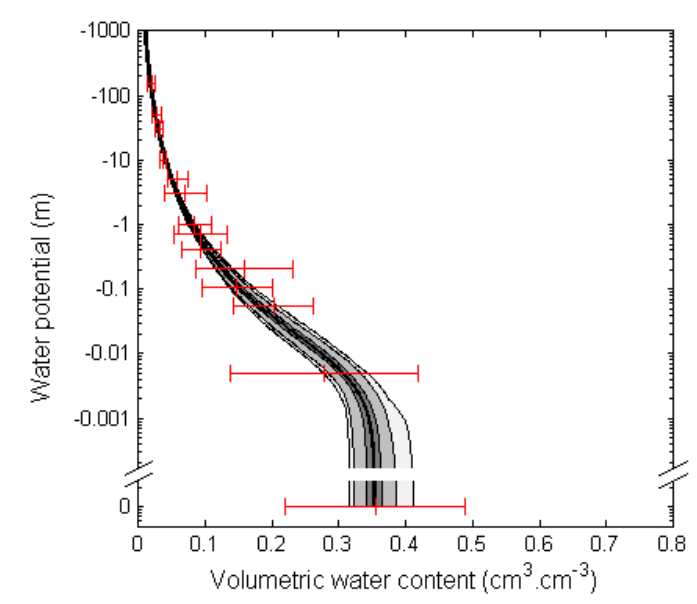

(a)

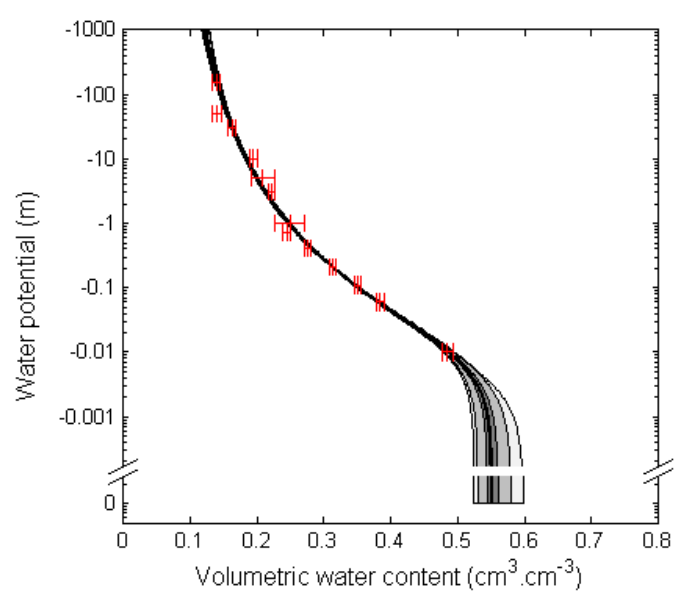

(c)

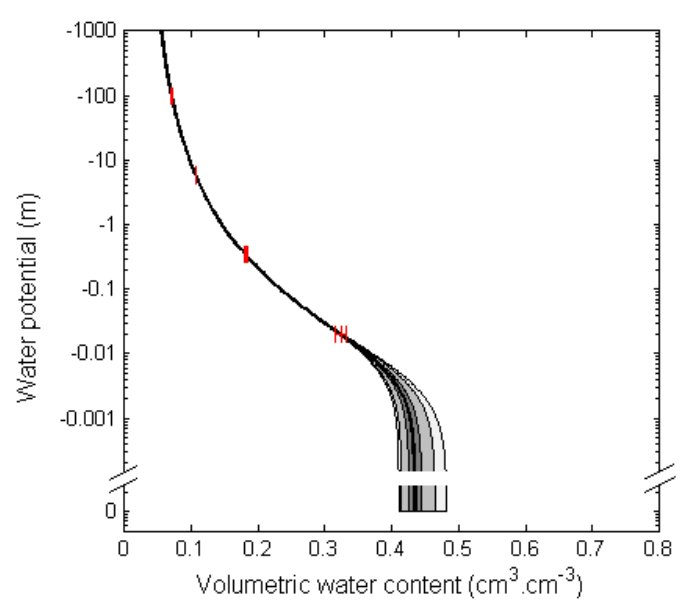

(e)

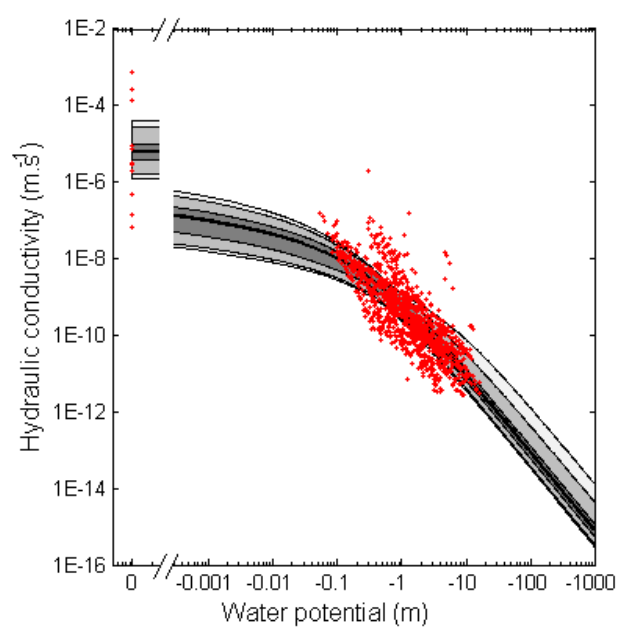

(b)

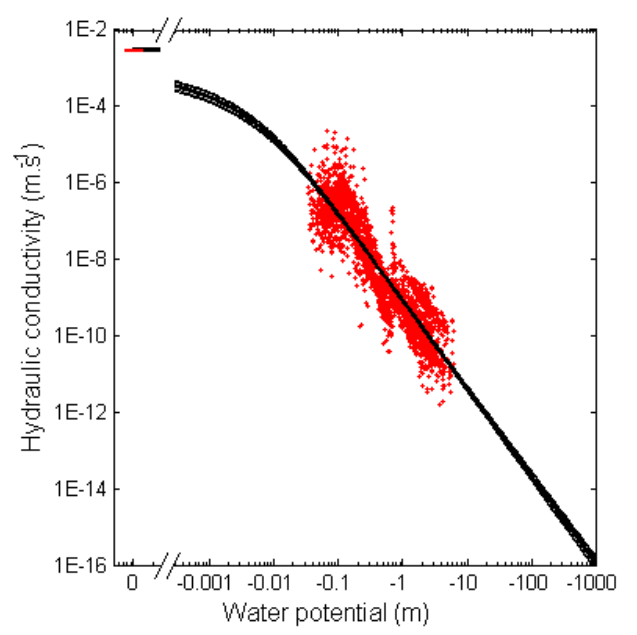

(d)

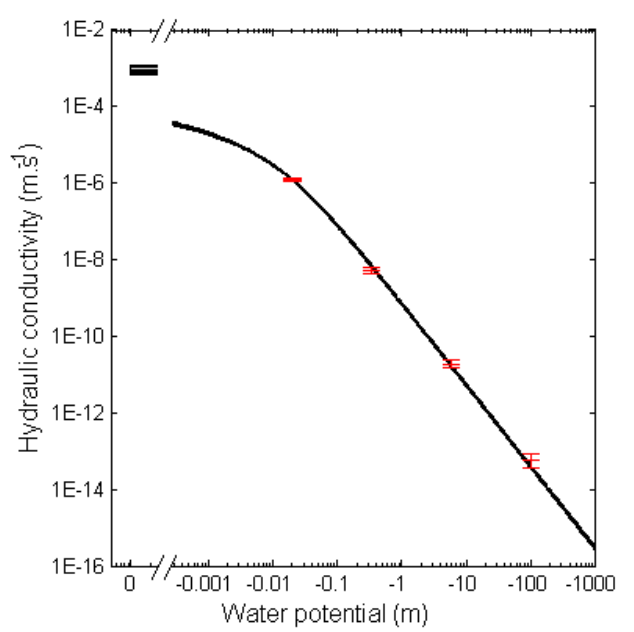

(f) 


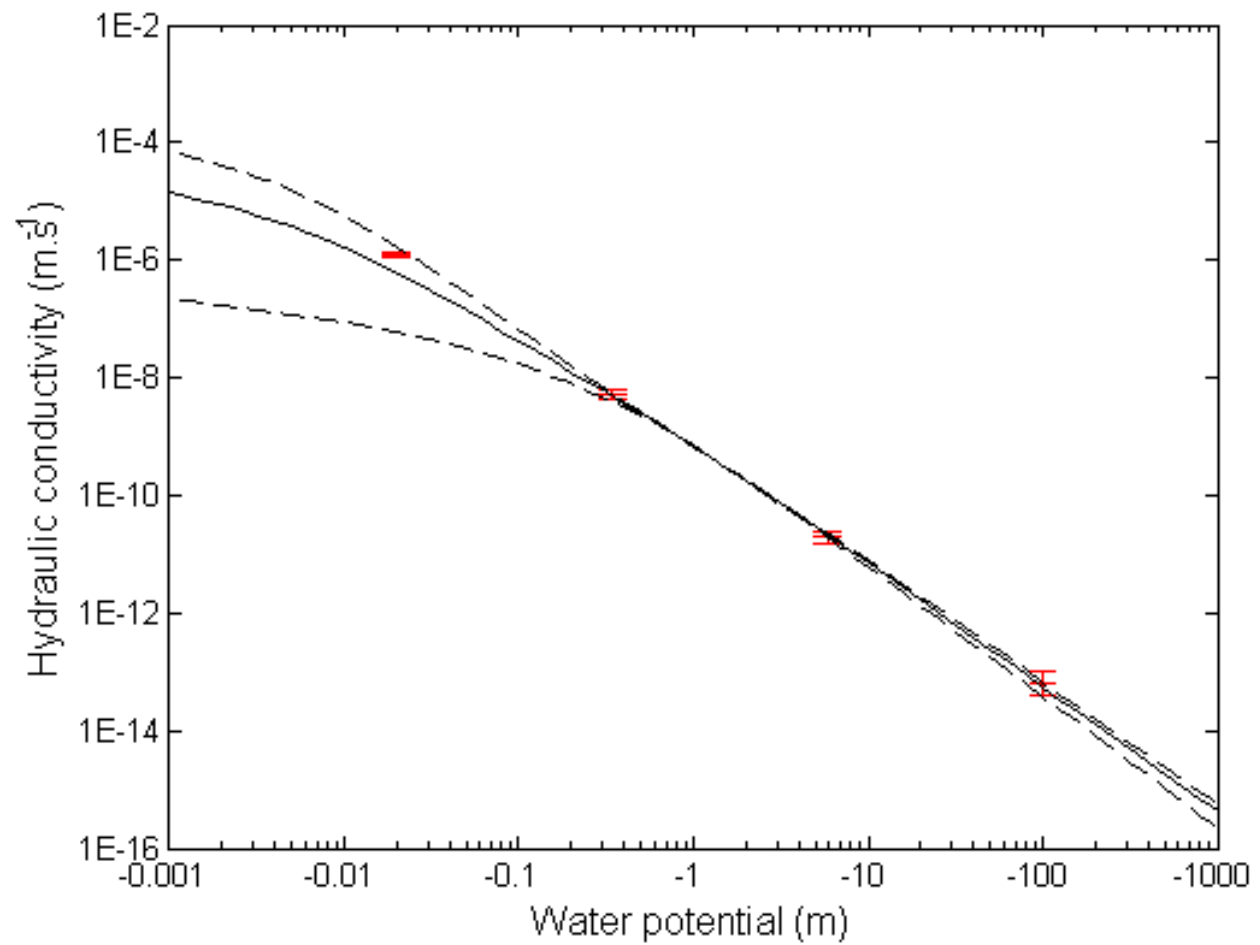

(a)

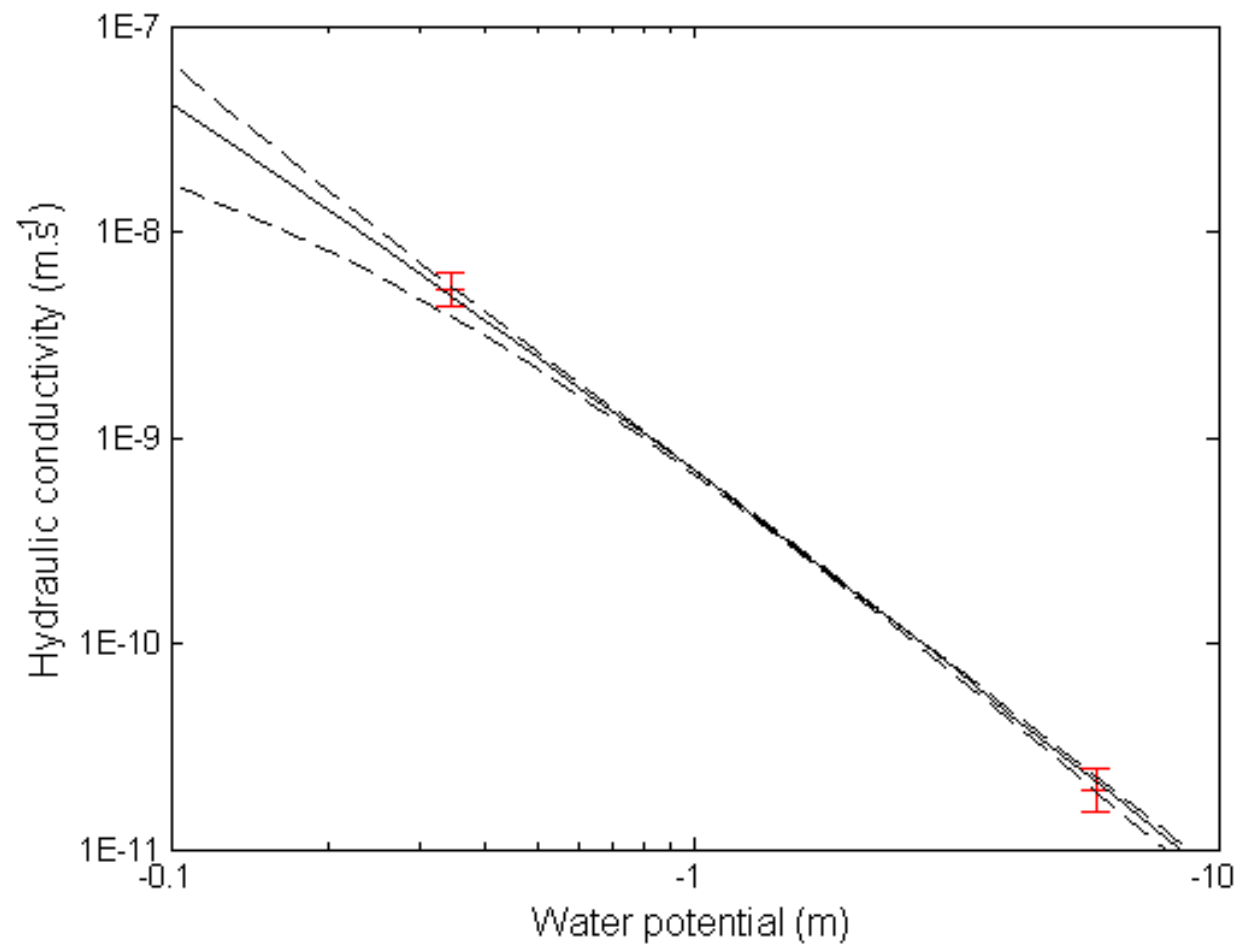

(b) 


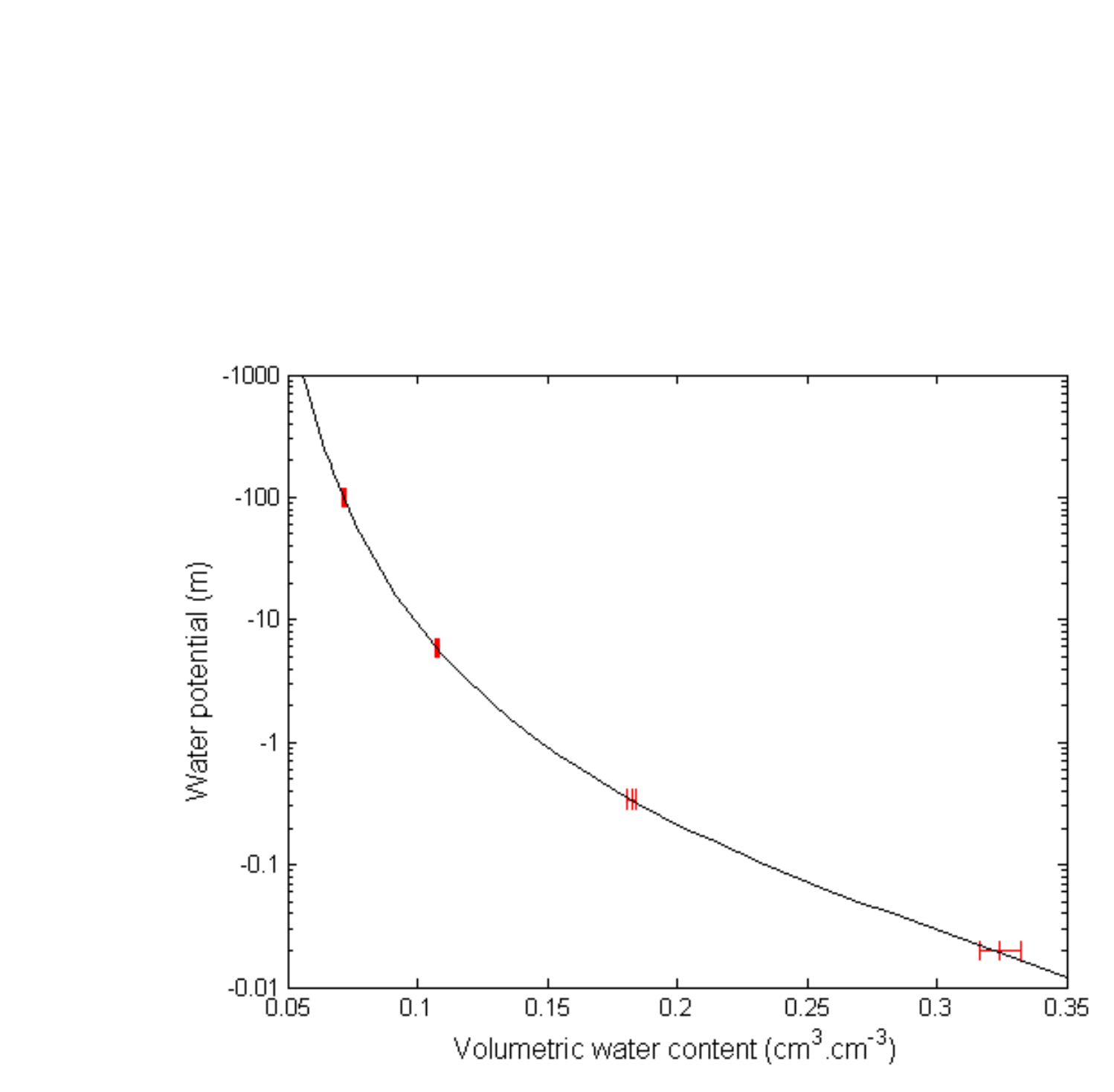

Figure 5

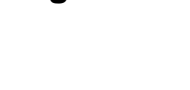
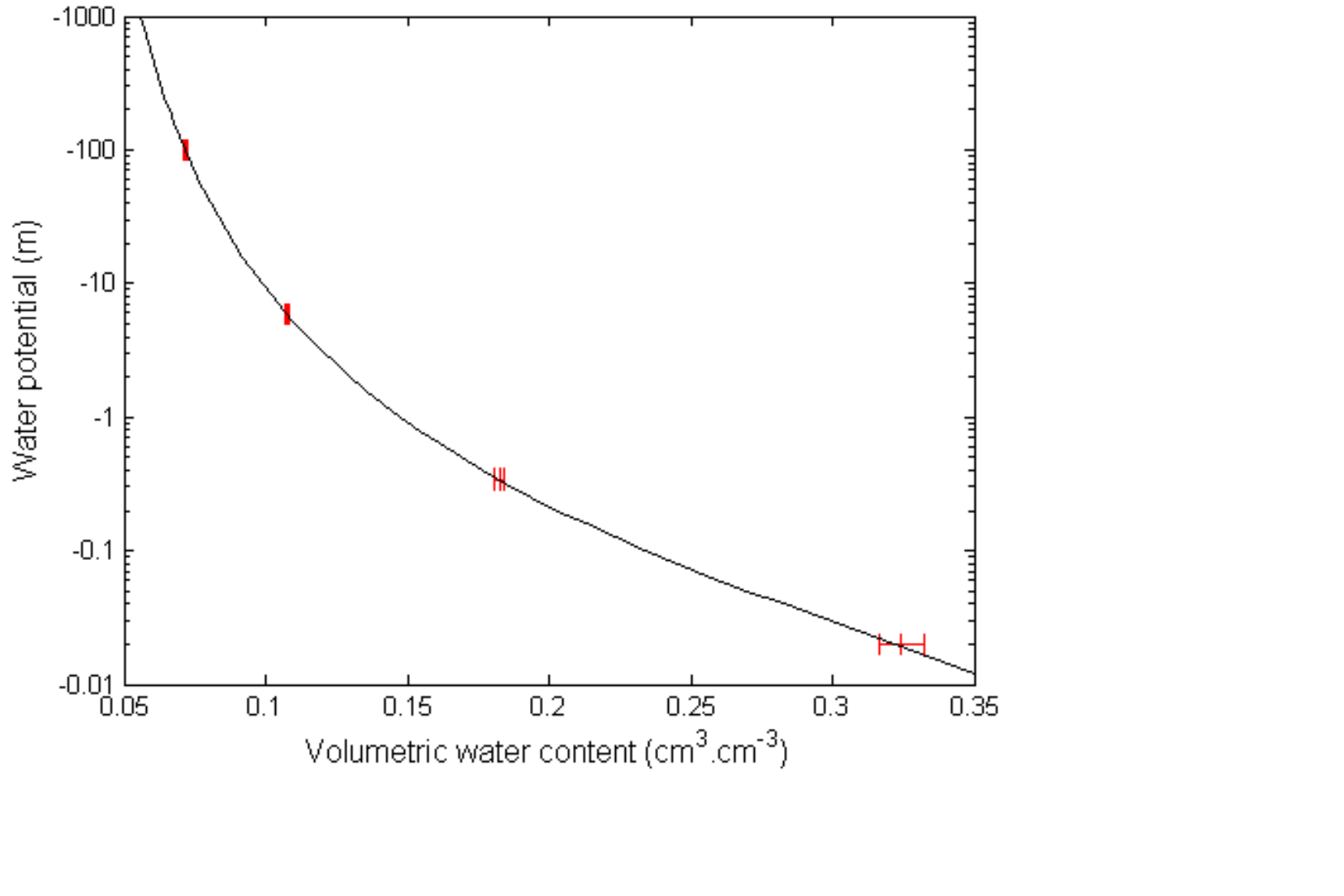


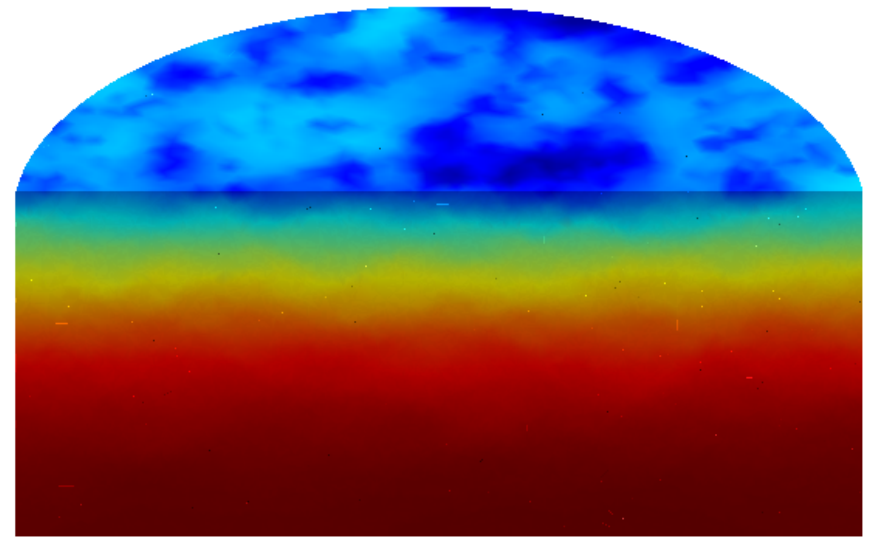

$-0.192$

$-0.169$

(a)

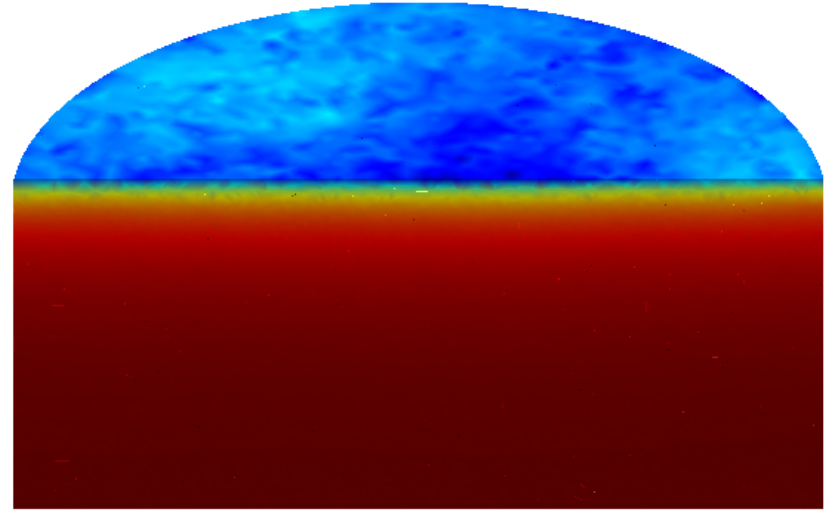

$-6.1$

$-0.971$

(b)

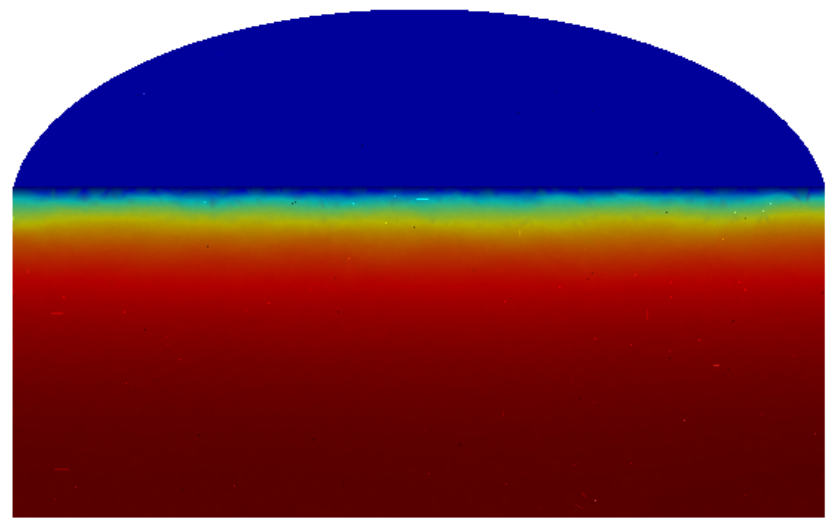

$-57.5$

$-15.1$

(c)

\section{)}


Figure 7

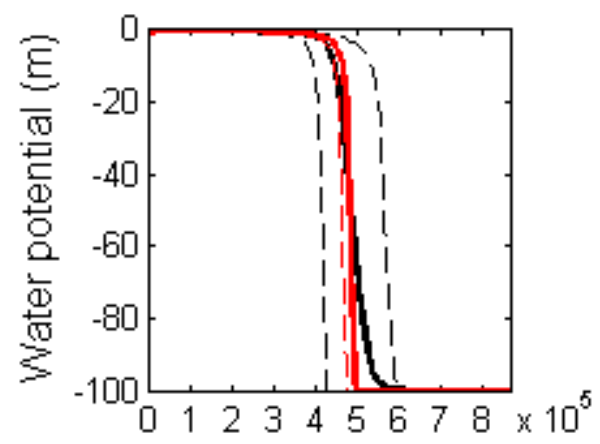

(a)

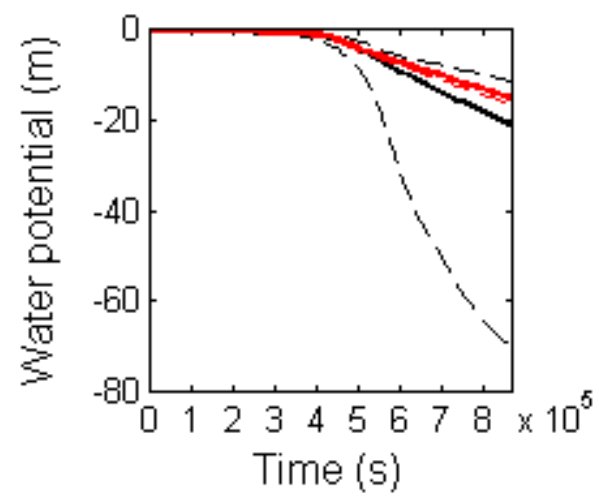

(c)

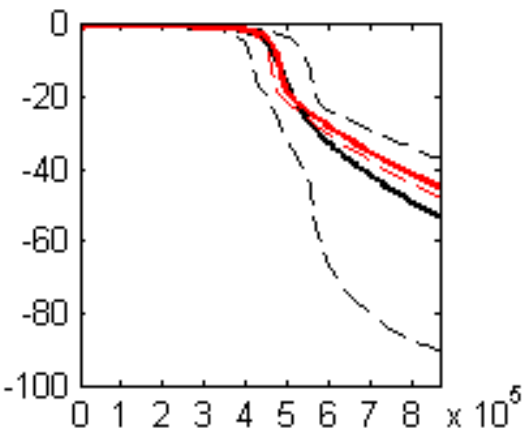

(b)

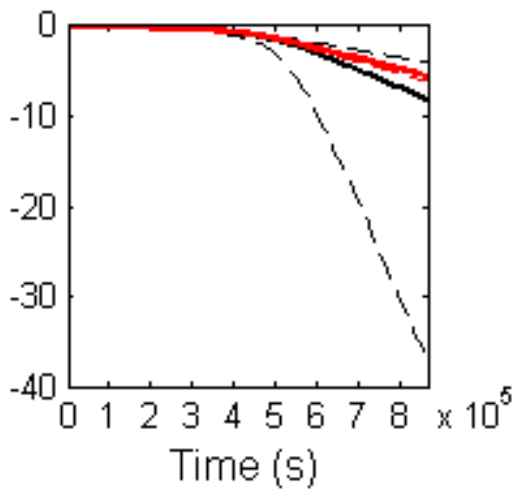

(d) 